\title{
A new algorithm for computing the Geronimus transformation with large shifts
}

\author{
Maria Isabel Bueno Cachadina • Alfredo Deaño • \\ Edward Tavernetti
}

Received: 11 December 2008 / Accepted: 29 July 2009 /

Published online: 8 August 2009

(C) The Author(s) 2009. This article is published with open access at Springerlink.com

\begin{abstract}
A monic Jacobi matrix is a tridiagonal matrix which contains the parameters of the three-term recurrence relation satisfied by the sequence of monic polynomials orthogonal with respect to a measure. The basic Geronimus transformation with shift $\alpha$ transforms the monic Jacobi matrix associated with a measure $d \mu$ into the monic Jacobi matrix associated with $d \mu /(x-\alpha)+$ $C \delta(x-\alpha)$, for some constant $C$. In this paper we examine the algorithms available to compute this transformation and we propose a more accurate algorithm, estimate its forward errors, and prove that it is forward stable. In particular, we show that for $C=0$ the problem is very ill-conditioned, and we present a new algorithm that uses extended precision.
\end{abstract}

Keywords Geronimus transformation - Accuracy • Roundoff error analysis • Orthogonal polynomials $\cdot$ Three-term recurrence relations

\footnotetext{
The first author's work was supported by Dirección General de Investigación (Ministerio de Ciencia y Tecnología) of Spain under grant MTM2006-06671. The third author's work was funded by EAF during the UCSB Summer Research Program for Undergraduates in 2007. A. Deaño acknowledges financial support from the Spanish Ministry of Education and Science, under the program of postdoctoral grants (Programa de becas postdoctorales) and project MTM2006-09050.
}

M. I. Bueno Cachadina $(\bowtie)$

Department of Mathematics and College of Creative Studies, University of California, Santa Barbara, CA, USA

e-mail: mbueno@math.ucsb.edu

A. Deaño

DAMTP, Centre for Mathematical Sciences, University of Cambridge, Cambridge, UK e-mail:ad495@cam.ac.uk

E. Tavernetti

Department of Mathematics, University of California, Davis, CA, USA

e-mail: etavernetti@math.ucdavis.edu 
Mathematics Subject Classifications (2000) $15 \mathrm{~A} 21 \cdot 15 \mathrm{~A} 23 \cdot 05 \mathrm{~A} 05 \cdot 05 \mathrm{~B} 25$

\section{Introduction}

Given a measure $\mu$, with supp $\mu \subset \mathbb{R}$, one can define a linear functional $\mathscr{L}$ on the space $\mathbb{P}$ of polynomials with real coefficients in the following way:

$$
\mathscr{L}(p)=\int p(x) d \mu(x), \quad p \in \mathbb{P},
$$

which is well defined provided that the moments $\mathscr{L}_{n}:=\mathscr{L}\left(x^{n}\right)$ are finite, $n=0,1,2, \ldots$ In that case, we say that $\mathscr{L}$ is a moment functional. Moreover, if the leading principal submatrices of the Hankel matrix $M=\left(\mathscr{L}_{i+j}\right)_{i, j=0}^{\infty}$ are nonsingular, then $\mathscr{L}$ is said to be quasi-definite, and there exists a sequence of polynomials $\left\{P_{n}\right\}_{n=0}^{\infty}$ orthogonal with respect to $\mu$, that is, [4]

1. $\operatorname{deg}\left(P_{n}\right)=n$ for all $n \geq 0$.

2. $\mathscr{L}\left(P_{n} P_{m}\right)=K_{n} \delta_{n, m}$, where $K_{n} \neq 0$ and $\delta_{n, m}$ is the "Kronecker delta" defined by

$$
\delta_{n, m}=\left\{\begin{array}{l}
0, \text { if } m \neq n, \\
1, \text { if } m=n .
\end{array}\right.
$$

In particular, $\left\{P_{n}\right\}_{n=0}^{\infty}$ is said to be a monic sequence of orthogonal polynomials (MOPS) if the leading coefficient of each polynomial is equal to one. Every MOPS satisfies a three-term recurrence relation (TTRR):

$$
x P_{n}(x)=P_{n+1}(x)+B_{n+1} P_{n}(x)+G_{n} P_{n-1}(x),
$$

$$
P_{-1}(x) \equiv 0, \quad P_{0}(x) \equiv 1, \quad B_{n}, G_{n} \in \mathbb{R}, \quad G_{0}=\mathscr{L}_{0}, \quad G_{n} \neq 0 \quad \text { for all } n \geq 0 .
$$

The previous set of equations can be written in matrix notation as

$$
x p=J p,
$$

where $p=\left[P_{0}(x), P_{1}(x), P_{2}(x), \ldots\right]^{T}$ and

$$
J=\left[\begin{array}{cccc}
B_{1} & 1 & 0 & \ldots \\
G_{1} & B_{2} & 1 & \ldots \\
0 & G_{2} & B_{3} & \ldots \\
\vdots & \vdots & \vdots & \ddots
\end{array}\right] .
$$

This semi-infinite tridiagonal matrix $J$ is called the monic Jacobi matrix associated with the functional $\mathscr{L}$. It is very unusual to denote the entries of a matrix by capital letters, but since the algorithms to compute the Geronimus transformation involve two monic Jacobi matrices, for the sake of clarity, we denote by capital letters the entries in the input matrix and by the same lowercase letters the entries in the output matrix. 
For a moment functional $\mathscr{L}$, a polynomial $\pi$, and a real number $\alpha$, let $\pi \mathscr{L}$ and $(x-\alpha)^{-1} \mathscr{L}$ be the moment functionals defined by

$$
\begin{aligned}
(\pi \mathscr{L})(p) & =\mathscr{L}(\pi p) \\
\left((x-\alpha)^{-1} \mathscr{L}\right)(p) & =\mathscr{L}\left(\frac{p(x)-p(\alpha)}{x-\alpha}\right) .
\end{aligned}
$$

In the literature there are numerous results studying the connection between the recurrence relations of polynomials orthogonal with respect to two allied measures $[1,2,7,15,24]$. This relationship can be extended to the corresponding Jacobi matrices. Two examples stand out as particularly important:

- Given $\mathscr{L}$ and $\alpha \in \mathbb{R}$, the transformation that gives the monic Jacobi matrix associated with $(x-\alpha) \mathscr{L}$ in terms of the monic Jacobi matrix associated with $\mathscr{L}$ is called the Christoffel transformation or Darboux transformation.

- Given $\mathscr{L}$, we consider the linear functional $\mathscr{G}:=(x-\alpha)^{-1} \mathscr{L}+M \delta(x-$ $\alpha$ ), where $\alpha \in \mathbb{R}$ is out of the support of the measure that defines $\mathscr{L}$, and $M$ is a nonzero constant. This transformation performs a rational modification of the measure that defines the functional $\mathscr{L}$ and add a Dirac mass in $\alpha$. Notice that $M=\mathscr{G}_{0}$, the first moment of $\mathscr{G}$. The transformation that gives the monic Jacobi matrix associated with $\mathscr{G}$ in terms of the monic Jacobi matrix associated with $\mathscr{L}$ is called the Geronimus transformation or Darboux transformation with free parameter.

These transformations can be considered as reciprocal in the following sense:

Lemma 1 [25] Let $\mathscr{L}$ and $\mathscr{G}$ be two linear functionals and $\alpha$ a real number. Then,

$$
(x-\alpha) \mathscr{G}=\mathscr{L} \quad \text { if and only if } \quad \mathscr{G}=(x-\alpha)^{-1} \mathscr{L}+\mathscr{G}_{0} \delta(x-\alpha) .
$$

If the functional $\mathscr{L}$ is expressed in integral form as in (1), then

$$
\mathscr{G}(p(x))=\left[(x-\alpha)^{-1} \mathscr{L}+\mathscr{G}_{0} \delta(x-\alpha)\right](p(x))=\int p(x) \frac{d \mu}{x-\alpha}+C p(\alpha),
$$

where $C=\mathscr{G}_{0}-\mu_{0}$ and $\mu_{0}=\int \frac{d \mu}{x-\alpha}$. Therefore, this transformation depends on two free parameters $\alpha$ and $C$. From now on we call the transformation that gives the monic Jacobi matrix associated with the functional $\mathscr{G}$ in terms of the monic Jacobi matrix associated with $\mathscr{L}$ the Geronimus transformation with shift $\alpha$ and parameter $C$.

The Geronimus transformation was first studied by Geronimus in 1940. Among numerous papers by Geronimus on orthogonal polynomials there are two $[13,14]$ which contain ideas that anticipated many investigations in modern mathematical physics. The main contribution by Geronimus was a deep investigation of both Darboux transformations. The first non-trivial 
application of these transformations was proposed by Geronimus himself in [13]. This application is connected to the problem of classifying all sequences of orthogonal polynomials such that its derivatives form another set of orthogonal polynomials. In the last two decades, these transformations have attracted the interest of various specialists in different branches of mathematics and mathematical physics for their applications to different topics such as Discrete Integrable Systems [20, 22, 23], Quantum Mechanics, Bispectral Transformations in Orthogonal Polynomials [16-18], and Numerical Analysis $[5,7,8,10,12]$.

The problem of the numerical computation of the Geronimus transformation with shift $\alpha$ and parameter $C$ of a Jacobi matrix $J$ has been extensively studied when $C=0$ and the shift $\alpha$ is close to the support of the measure $\mu$ $[5,8,10]$. However, we have not found any papers on the case $C \neq 0$, or when $C=0$ and the shift is not close to the support of the measure.

The objectives of this paper are the following:

- to investigate the numerical behavior of the available algorithms to compute the Geronimus transformation,

- to present a new algorithm which is more accurate than the previous ones (Algorithm 3, specially when the shift moves away from the support of the measure,

- to explain why the numerical behavior of any algorithm to compute the Geronimus transformation is considerably different for $C=0$ and $C \neq 0$.

We also estimate the forward errors (Theorem 2) produced by the new algorithm with $\mathcal{O}(n)$ cost, and prove that this algorithm is componentwise forward stable (Theorem 8), which means that the magnitude of the errors produced by this algorithm are the best ones that can be expected because they reflect the sensitivity of the problem to perturbations in the input data (Theorem 2). No need to say that forward stability does not imply small forward errors when the problem is ill-conditioned.

We also show that this algorithm is more accurate than the previous ones, specially when the shift moves away from the support of the measure and $C \neq 0$ (Section 4.4). We prove that the problem of computing the Geronimus transformation is extremely ill-conditioned when $C=0$ (Subsection 4.5) and therefore, a significant loss of accuracy can be expected in this case. However, we also show that by computing a few outputs with extended precision, the algorithm becomes much more accurate (See Table 7).

The paper is structured as follows: in Section 2 we give a brief account of the main theoretical results needed. In Section 3 we analyze the available forward and backward algorithms, and in Section 4 we introduce a new algorithm. We present a backward error analysis of this algorithm (Theorem 4.1) and provide a condition number for the problem (Definition 2 and Theorem 3 ) that allows us to estimate the forward errors produced by the new algorithm in $\mathcal{O}(n)$ flops. Finally, we show several numerical experiments to illustrate the performance of this new method and we prove that it is componentwise forward stable. 


\section{Theoretical results on the Geronimus transformation}

Throughout this section, $\mathscr{L}$ is a quasi-definite moment functional, $\left\{P_{n}\right\}$ the sequence of monic polynomials orthogonal with respect to $\mathscr{L}, J$ the monic Jacobi matrix associated with $\left\{P_{n}\right\}$, and $\alpha$ a real number outside the support of the measure that defines $\mathscr{L}$.

Let $J-\alpha I=U L$ denote a decomposition of $J-\alpha I$ as a product of an upper triangular matrix $U$ and a unit lower triangular matrix $L$, where

$$
U=\left(\begin{array}{cccc}
u_{1} & 1 & 0 & \ldots \\
0 & u_{2} & 1 & \ldots \\
0 & 0 & u_{3} & \ldots \\
\vdots & \vdots & \vdots & \ddots
\end{array}\right), L=\left(\begin{array}{cccc}
1 & 0 & 0 & \ldots \\
l_{1} & 1 & 0 & \ldots \\
0 & l_{2} & 1 & \ldots \\
\vdots & \vdots & \vdots & \ddots
\end{array}\right),
$$

whenever it is possible. It is easy to check that whenever the $U L$ factorization of $J-\alpha I$ exists, it is not unique. In fact, the entry $u_{1}$ can be considered a free parameter. Then, given $\alpha$ and $u_{1}$, we say that $\tilde{J}=L U+\alpha I$ is the Geronimus transform of $J$ with shift $\alpha$ and parameter $u_{1}$.

Necessary and sufficient conditions for the existence of the Geronimus transform with shift $\alpha$ and parameter $u_{1}$ of a monic Jacobi matrix $J$ are given in [1] and [25].

It is also clear that $\tilde{J}$ is a tridiagonal semi-infinite matrix. By Favard's theorem [4], $\tilde{J}$ generates a new sequence of monic orthogonal polynomials if and only if the entries of $\tilde{J}$ in positions $(i+1, i)$ for $i \geq 1$ are all nonzero. In this case, the MOPS associated with $J$ and $\tilde{J}$, respectively, can be related through the matrix $L$, as we next show.

Lemma 2 Let $J$ be a monic Jacobi matrix and let $\alpha \in \mathbb{R}$ be such that $J-\alpha I$ has an UL factorization. Let $u_{1} \in \mathbb{R}$ and let $\tilde{J}$ be the Geronimus transform with shift $\alpha$ and parameter $u_{1}$ of J. Assume that $\left\{P_{n}\right\}$ and $\left\{Q_{n}\right\}$ are, respectively, the MOPS associated with $J$ and $\tilde{J}$. If $J-\alpha I=U L$ is the $U L$ factorization of $J-\alpha I$ such that $\tilde{J}=L U+\alpha I$, then $L$ is the change of basis matrix from $\left\{P_{n}\right\}$ to $\left\{Q_{n}\right\}$, i.e. $Q=L P$, where $Q$ and $P$ are, respectively, the column vectors containing the polynomials in $\left\{P_{n}\right\}$ and $\left\{Q_{n}\right\}$.

Proof Multiply $J-\alpha I=U L$ by $L$ on the left to get

$$
L(J-\alpha I)=(L U) L .
$$

Replace $L U$ by $\tilde{J}-\alpha I$ in (4) and multiply by $L^{-1}$ on the right to get

$$
L(J-\alpha I) L^{-1}=\tilde{J}-\alpha I
$$

Thus, $J-\alpha I$ is similar to $\tilde{J}-\alpha I$. Considering the relation $x Q=\tilde{J} Q$, we have

$$
(x-\alpha) Q=(\tilde{J}-\alpha I) Q=L(J-\alpha I) L^{-1} Q
$$

and multiplying by $L^{-1}$ on the left we have

$$
(x-\alpha) L^{-1} Q=(J-\alpha I) L^{-1} Q
$$


and hence $x\left(L^{-1} Q\right)=J\left(L^{-1} Q\right)$, and $L^{-1} Q$ is a MOPS $p$ satisfying $x p=J p$. By uniqueness $L^{-1} Q=P$, which implies the result.

It can be proven $[1,25]$ that if the matrix $J-\alpha I=U L$, with $U$ and $L$ as in (3), then the Geronimus transform with shift $\alpha$ and parameter $u_{1}$ is the Jacobi matrix associated with a functional $\mathscr{G}$ given by

$$
\mathscr{G}=(x-\alpha)^{-1} \mathscr{L}+\mathscr{G}_{0} \delta(x-\alpha),
$$

where $\mathscr{G}_{0}$ is the first moment of the functional $\mathscr{G}$. Next we show the relationship between $\mathscr{G}_{0}$ and the parameter $u_{1}$ involved in the $U L$ factorization of $J$.

Lemma 3 Let $\mathscr{L}$ be a quasi-definite moment functional, and $J$ the corresponding Jacobi matrix. Then, the Geronimus transform of $J$ with shift $\alpha$ and parameter $u_{1}$ is associated with the moment functional

$$
\mathscr{G}=(x-\alpha)^{-1} \mathscr{L}+\frac{\mathscr{L}_{0}}{u_{1}} \delta(x-\alpha),
$$

where $\mathscr{L}_{0}$ is the first moment of the functional $\mathscr{L}$. Moreover, if the integral representation of $\mathscr{L}$ is given by

$$
\mathscr{L}(p)=\int p(x) d \mu(x),
$$

then the Geronimus transform of $J$ is associated with the moment functional with integral representation

$$
\mathscr{G}(p)=\int p(x) \frac{d \mu(x)}{x-\alpha}+\left(\frac{\mathscr{L}_{0}}{u_{1}}-\mu_{0}\right) p(\alpha),
$$

where $\mu_{0}=\int \frac{d \mu(x)}{x-\alpha}$ and $p \in \mathbb{P}$.

Proof By Lemma 1, $(x-\alpha) \mathscr{G}=\mathscr{L}$. Let $\left\{P_{n}\right\}_{n=0}^{\infty}$ and $\left\{Q_{n}\right\}_{n=0}^{\infty}$ be the MOPS with respect to $\mathscr{L}$ and $\mathscr{G}$, respectively. Then, if we denote $P=$ $\left[P_{1}(x), P_{2}(x), \ldots .\right]^{t}$, and $Q=\left[Q_{1}(x), Q_{2}(x), \ldots . .\right]^{t}$, we get

$$
((x-\alpha) \mathscr{G})\left(Q Q^{t}\right)=\mathscr{L}\left(Q Q^{t}\right) .
$$

Taking into account Lemma 2,

$$
\mathscr{G}\left((x-\alpha) Q Q^{t}\right)=\mathscr{L}\left(L P P^{t} L^{t}\right) .
$$

Considering the recurrence relation that $\left\{Q_{n}\right\}$ satisfies and the linearity of $\mathscr{L}$ and $\mathscr{G}$

$$
\begin{aligned}
\mathscr{G}\left((\tilde{J}-\alpha I) Q Q^{t}\right) & =L \mathscr{L}\left(P P^{t}\right) L^{t} \\
(\tilde{J}-\alpha I) \mathscr{G}\left(Q Q^{t}\right) & =L D_{p} L^{t}
\end{aligned}
$$


where $D_{p}$ is the diagonal matrix whose diagonal elements are given by $\left(D_{p}\right)_{i i}=\mathscr{L}\left(P_{i}^{2}\right)$ for all $i$. Thus,

$$
(\tilde{J}-\alpha I)=L\left(D_{p} L^{t} D_{q}^{-1}\right)=L U,
$$

where $D_{q}$ is defined similarly to $D_{p}$. Notice that $D_{p}$ and $D_{q}$ are invertible matrices by definition of orthogonal polynomials.

Finally, this implies that $u_{1}=\mathscr{L}_{0} / \mathscr{G}_{0}$, and the result follows. The last part of the lemma is obtained by considering the integral representation of $\mathscr{L}$, that is,

$$
\mathscr{G}(p)=\int p(x) \frac{d \mu(x)}{x-\alpha}-p(\alpha) \int \frac{d \mu(x)}{x-\alpha}+\frac{\mathscr{L}_{0}}{u_{1}} p(\alpha) .
$$

From the point of view of the algorithms that we will present in the next section, we need to use the fact that the MOPS $\left\{P_{n}(x)\right\}_{n=0}^{\infty}$ obeys a three term recurrence relation of the form:

$$
y_{n+1}=\left(\alpha-B_{n+1}\right) y_{n}-G_{n} y_{n-1}, \quad n \geq 0 .
$$

Now it is important to note that if $\alpha \notin \operatorname{supp} \mu$, then the functions $\left\{\rho_{n}(\alpha, C)\right\}_{n=-1}^{\infty}$ defined by

$\rho_{n}(\alpha, C)=-\mathscr{G}\left(P_{n}(x)\right)=-\left(\int P_{n}(x) \frac{d \mu}{x-\alpha}+C P_{n}(\alpha)\right), \quad n \geq 0, \quad \rho_{-1}(\alpha, C)=1$

obey the same TTRR satisfied by the sequence of orthogonal polynomials $P_{n}(x)$ for every value of $C$. Moreover, when $C=0$ it turns out that $\left\{\rho_{n}(\alpha, C)\right\}_{n=-1}^{\infty}$ is the minimal solution of this recursion, which means that

$$
\lim _{n \rightarrow \infty} \frac{\rho_{n}(\alpha, 0)}{g_{n}}=0,
$$

for any other solution of the TTRR, say $g_{n}$, which is independent of $\rho_{n}(\alpha, 0)$. The solution $g_{n}$ is called dominant, see [10] for more details on the general theory.

As a consequence of this situation, when $C=0$, it is not recommended to use the three-term recurrence relation in the forward direction (for increasing $n)$ to generate $\left\{\rho_{n}(\alpha, C)\right\}_{n=-1}^{\infty}$, due to numerical instability. However, the TTRR can be used in the backward direction, and the process can be reformulated in terms of the associated continued fraction

$$
\frac{y_{n}}{y_{n-1}}=\frac{G_{n}}{\alpha-B_{n+1}-} \frac{G_{n+1}}{\alpha-B_{n+2}-\alpha-B_{n+3}-} \frac{G_{n+2}}{\alpha, \quad n=0,1,2, \ldots}
$$

which converges to the ratio of minimal solutions according to Pincherle's theorem [11]. 
Let us define the following quantities:

$$
r_{n-1}:=\frac{\rho_{n}(\alpha, C)}{\rho_{n-1}(\alpha, C)}=\frac{G_{n}}{\alpha-B_{n+1}-} \frac{G_{n+1}}{\alpha-B_{n+2}-} \frac{G_{n+2}}{\alpha-B_{n+3}-} \ldots, \quad n=0,1,2, \ldots
$$

Note that, in particular, $r_{-1}=\rho_{0}(\alpha, C)=-\left(\mu_{0}+C\right)$. The importance of these variables in the Geronimus transformation will be given in Lemma 4 , which expresses the quantities $r_{k}$ defined in (6) in terms of the entries in the subdiagonal of the matrix $L$ in the $U L$ factorization of $J-\alpha I$.

Lemma 4 Let $\left\{P_{n}\right\}$ be the sequence of monic polynomials orthogonal with respect to the linear functional $\mathscr{L}(p)=\int p d \mu$. Let $C, \alpha \in \mathbb{R}$, and $\alpha \notin \operatorname{supp} \mu$. Assume that $J-\alpha I=U L$ is the $U L$ factorization of $J-\alpha I$ such that $\tilde{J}=L U+$ $\alpha I$ is the monic Jacobi matrix associated with $\mathscr{G}(p)=\int p(x) /(x-\alpha) d \mu+$ $C p(\alpha)$. Then,

$$
r_{k-1}:=\frac{\rho_{k}(\alpha, C)}{\rho_{k-1}(\alpha, C)}=-l_{k}, \quad \text { for all } k \geq 1,
$$

where $l_{k}=L(k+1, k)$.

Proof The result can be proven by induction. After dividing by $\rho_{k-1}(\alpha, C)$ the TTRR

$$
\rho_{k}(\alpha, C)=\left(\alpha-B_{k}\right) \rho_{k-1}(\alpha, C)-G_{k-1} \rho_{k-2}(\alpha, C), \quad k \geq 1,
$$

consider the expression for $l_{k}$ given in Algorithm 1.

\section{Algorithms for computing the Geronimus transformation and numerical experiments}

In this section we examine the currently available algorithms for numerically generating a Geronimus transform of a monic Jacobi matrix $J$. First we present the standard algorithm which can be derived from the matrix version of the Geronimus transformation given in (8). Then, we present other algorithms used in the literature.

When $C=0$ and the shift $\alpha$ is close to the support of the measure, researchers $[5,10]$ recommend a split strategy, that is, to use a "forward algorithm" when the shift $\alpha$ approaches the support of the measure, and a "backward algorithm" when the shift moves away from the support.

When $C \neq 0$, we can still use the "forward algorithms". However, the "backward algorithm" does not converge and is not useful as we explain below. In this section, we also show, through numerical experiments, that the "forward algorithms" and the "backward algorithm" (when available) become less accurate as the shift moves away from the support of the measure.

From now on all the results refer to leading principal submatrices of monic Jacobi matrices. Since we are interested in the numerical analysis of algorithms 
that implement the Geronimus transformation, we can only consider finite matrices. We denote by $J_{n}(B, G)$ the $n \times n$ leading principal submatrix of $J$, where $B=\left[B_{1}, \ldots, B_{n}\right]^{T}$, and $G=\left[G_{1}, \ldots, G_{n-1}\right]^{T}$ and $\tilde{J}_{n}(b, g)$ is the $n \times n$ leading principal submatrix of $\tilde{J}, b=\left[b_{1}, \ldots, b_{n}\right]^{T}$ being the elements on the main diagonal of $\tilde{J}_{n}(b, g)$, and $g=\left[g_{1}, \ldots, g_{n-1}\right]^{T}$ the elements on the first lower subdiagonal. Then, the finite version of the Geronimus transformation with shift $\alpha$ and parameter $u_{1}$ is given by

$$
J_{n}(B, G)-\alpha I_{n}=U_{n} L_{n}+l_{n} e_{n} e_{n}^{t}, \quad \tilde{J}_{n}(b, g)=L_{n} U_{n}+\alpha I_{n},
$$

where $e_{n}$ denotes the $n$-th column of the $n$-by- $n$ identity matrix and $M_{n}$ denotes the leading principal submatrix of order $n$ of any matrix $M$.

Since we can only consider a finite leading principal submatrix of the initial monic Jacobi matrix as input for any algorithm to compute the Geronimus transformation, in order to determine the appropriate value of the free parameter $u_{1}$, the parameters $C, \mu_{0}$, and $\mathscr{L}_{0}$ need to be known (as Lemma 3 shows). Thus, in all the algorithms in this paper we consider as inputs $B, G, \alpha, C, \mu_{0}$, and $\mathscr{L}_{0}$.

The following pseudocode gives the standard algorithm to compute the Geronimus transform with shift $\alpha$ and parameters $C, \mu_{0}$, and $\mathscr{L}_{0}$ of an $n \times n$ monic Jacobi matrix $J_{n}(B, G)$. This algorithm is obtained from (8). Notice that

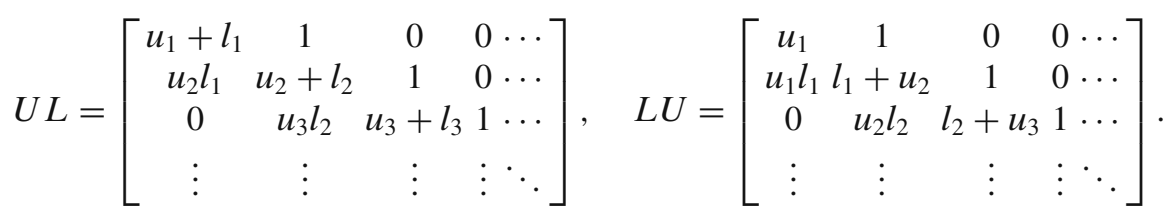

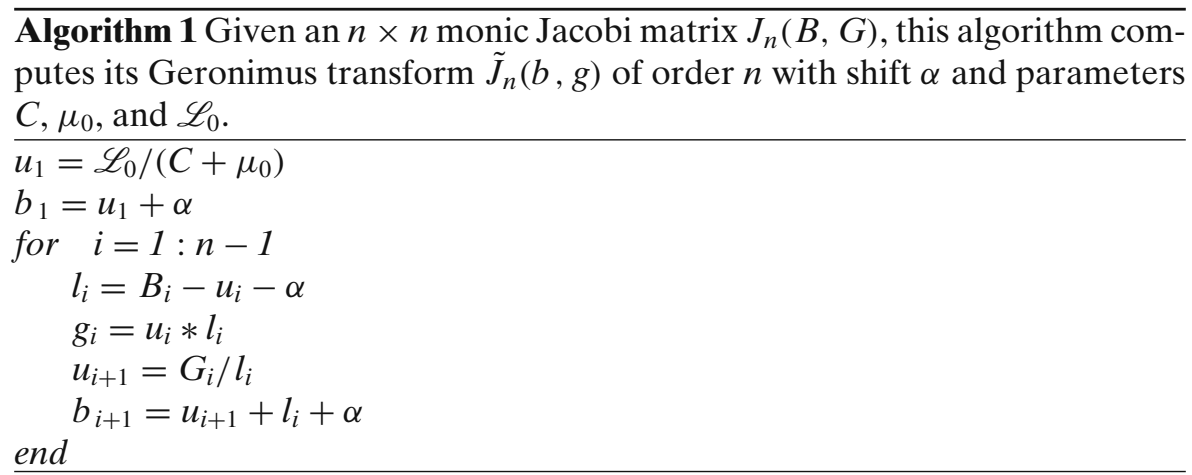


The computational cost of Algorithm 1 is $6 n-2$ flops. This algorithm is closely related to the qd-algorithm proposed by Rutishauser. In [21], Rutishauser introduces $\sigma$-degree monic polynomials $p_{\sigma}^{(v)}(x)$, depending on an additional integer parameter $v$ and with the initial condition $p_{0}^{(v)}(x) \equiv 1$. These polynomials satisfy two basic relations:

$$
p_{\sigma}^{(\nu)}(x)=x p_{\sigma-1}^{(\nu+1)}(x)-q_{\sigma}^{(\nu)} p_{\sigma-1}^{(\nu)}(x)
$$

and

$$
p_{\sigma}^{(v+1)}(x)-p_{\sigma}^{(v)}(x)=-e_{\sigma}^{(v)} p_{\sigma-1}^{(v+1)}(x) .
$$

Compatibility of these relations yields a three-term recurrence relation for the polynomials $p_{\sigma}^{(v)}$ (i.e. $p_{\sigma-1}^{(v+1)}$ are orthogonal polynomials), together with nonlinear relations for the coefficients $q_{\sigma}^{(v)}$ and $e_{\sigma}^{(v)}$ (i.e. the qd-algorithm). Relation (9) is equivalent to the Christoffel transformation from polynomials $p_{\sigma}^{(v)}(x)$ to polynomials $p_{\sigma}^{(v+1)}(x)$, while relation (10) is equivalent to Geronimus transformation from polynomials $p_{\sigma}^{(v+1)}(x)$ to polynomials $p_{\sigma}^{(v)}(x)$. These transformations are not shifted, that is, $\alpha=0$. However, already in the classical book [6, pg. 460], a generic scheme of the shifted Rutishauser algorithm is presented.

If we denote by $\left\{P_{n}\right\}$ and $\left\{Q_{n}\right\}$ the sequence of polynomials whose Jacobi matrix is $J$ and $\tilde{J}$, respectively, condition (10) is equivalent in our notation to

$$
P_{n}-Q_{n}=-l_{n} P_{n-1},
$$

(see (3) and Lemma 2).

Next we present an algorithm slightly different than Algorithm 1 that can be obtained by replacing $l_{k}$ by $-r_{k-1}$, using Lemma 4 , and eliminating the variables $u_{k}$ in Algorithm 1 .

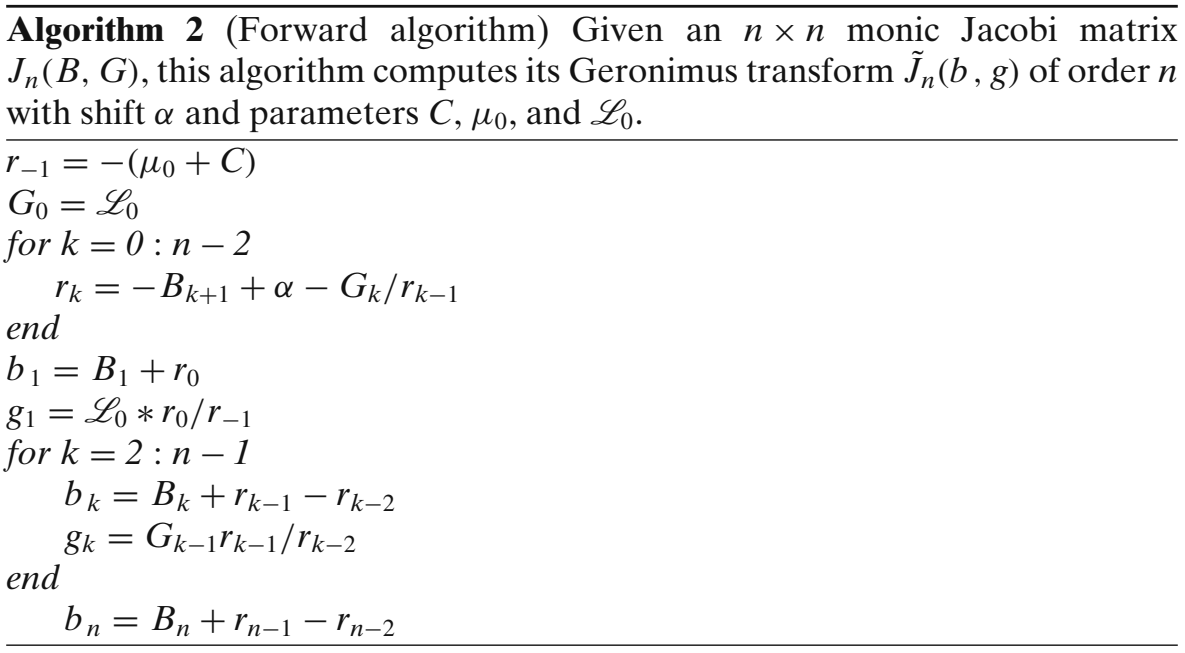

The computational cost of this algorithm is $7 n-3$ flops. 
Notice that both Algorithms 1 and 2 are "forward algorithms" since they compute $l_{n}$ and $r_{n}$, respectively, for increasing values of $n$. However we call Algorithm 2 "Forward Algorithm" because this is the algorithm proposed by W. Gautschi [10] in the split strategy for $C=0$.

W. Gautschi also proposes an alternative algorithm when $C=0$, in which the quantities $r_{k}$ are computed backwards. Namely, given an initial value $m \geq n$ :

$$
r_{m}=0, \quad r_{i-1}=\frac{G_{i}}{\alpha-B_{i+1}-r_{i}}, \quad i=m, m-1, \ldots, 1,
$$

together with $r_{-1}=\mathscr{L}_{0} /\left(\alpha-B_{1}-r_{0}\right)$. Observe that this is equivalent to (6). The quantities $b_{k}$ and $g_{k}$ are then computed in the same way as in the forward algorithm.

In [10] Gautschi studies the properties of Algorithm 2 and the backward method. He states that the forward algorithm is better when $\alpha$ is very close to the support of the measure and the order $n$ of $J_{n}(B, G)$ is not too large; otherwise, the backward algorithm is advised.

This backward algorithm can produce very accurate Jacobi matrices but, unlike the forward methods, it may require infeasibly large initial matrices $J_{m}(B, G)$ to produce an output matrix $\tilde{J}_{n}(b, g)$ of quite moderate dimension. Estimators for determining the advised initial order $m$ of $J_{m}(B, G)$ are given in [9] but they are only well-defined for the classical families of orthogonal polynomials.

Elhay and Kautsky [5] also suggest a split strategy in the case $C=0$, the backward algorithm being the same as the one proposed by Gautschi. However, the forward algorithm they propose, called the Inverse Cholesky algorithm, is more expensive than Algorithm 2 (computational cost of at least $O\left(n^{2}\right)$ ) and their numerical experiments in [5] show comparable performance.

\subsection{Numerical experiments}

Here we present some numerical experiments that show the accuracy of the algorithms presented in the previous subsection.

In order to check the accuracy of the algorithms, we have computed the following componentwise forward errors:

$$
\text { error } b=\max _{k=1 \ldots n}\left\{\left|\frac{b_{k}-\hat{b}_{k}}{b_{k}}\right|\right\}, \quad \text { error } g=\max _{k=1 \ldots n-1}\left\{\left|\frac{g_{k}-\hat{g}_{k}}{g_{k}}\right|\right\},
$$

where $\hat{b}_{k}$ and $\hat{g}_{k}$ denote the outputs computed by a given algorithm in standard double precision, i.e., $\mathbf{u} \approx 1.11 \times 10^{-16}$ is the unit roundoff of the finite arithmetic, while $b_{k}$ and $g_{k}$ denote the outputs obtained by running the same algorithm with 64 decimal digits of precision.

The experiments have been done using MATLAB 7.6.0 and the variable precision arithmetic of its Symbolic Math Toolbox. In all our tests, theoretical 
error bounds guarantee that the outputs obtained by running the algorithms with 64 decimal digits of precision have more than 50 significant decimal digits.

We have applied Algorithm 1, Algorithm 2 and the Backward Algorithm to the following Jacobi matrices:

1. The 60 -by-60 monic Jacobi matrix corresponding to the Jacobi polynomials with parameters $a=-1 / 3$ and $b=1 / 7$.

2. The 60-by-60 monic Jacobi matrix corresponding to the Laguerre polynomials with parameter $a=-1 / 3$.

In both cases, we considered a broad range of values for the shift $\alpha$ and two different values for the parameter $C=\{0,10\}$. For other nonzero values of $C$, the behavior of the algorithms is similar to that of $C=10$. The results can be found in Tables 1, 2, 3, and 4 .

Notice that when $C=0$, the three algorithms lose all their accuracy as the shift $\alpha$ moves away from the support. When $C \neq 0$, the accuracy of the algorithms also decreases as $\alpha$ moves away from the support although in a more moderate way. Notice that the numerical behavior of Algorithm 1 and the Forward Algorithm seems very similar.

\section{A new algorithm}

In this section we present a new algorithm to compute a Geronimus transform of a monic Jacobi matrix $J$. We will show that, with this new algorithm, the accuracy increases as $\alpha$ moves away from the support of the measure when $C \neq$ 0. In Section 4.6 we will also show that this new algorithm is forward stable. This means that the forward errors we get from this algorithm are the best that can be expected taking into account the conditioning of the problem.

This new algorithm does not improve the accuracy when $C=0$ because, as we will show in Subsection 4.3, the problem of computing the Geronimus transformation of a monic Jacobi matrix when $C=0$ is very ill-conditioned. We will also show that the conditioning of the problem depends strongly on the computation of the very first outputs and the accuracy increases notably when computing those outputs with extended accuracy and taking them as new inputs of the same algorithm.

The new algorithm that we present in this section only requires as input a monic Jacobi matrix of the same size as the output matrix. The numerical

Table 1 Algorithm 1-algorithm 2-backward algorithm

\begin{tabular}{lclclcl}
\hline$\alpha$ & Error $\mathbf{b}$ & Error $\mathbf{g}$ & Error $\mathbf{b}$ & Error $\mathbf{g}$ & Error $\mathbf{b}$ & Error $\mathbf{g}$ \\
\hline-1.0001 & $1.410^{-11}$ & $2.210^{-16}$ & $2.510^{-11}$ & $6.710^{-16}$ & $1.310^{-11}$ & $4.410^{-15}$ \\
-1.1 & 16.78 & 1.7 & 29.26 & 0.18 & 25 & 1.6 \\
-2 & 2.43 & 2.16 & 2.43 & 2.16 & 1.4 & 4.5 \\
-10 & 43.32 & 1.57 & 43.32 & 1.57 & 26.3 & 1.21 \\
\hline
\end{tabular}

Forward errors for Jacobi polynomials with $a=-1 / 3, b=1 / 7, n=60, C=0$ 
Table 2 Algorithm 1-algorithm 2

\begin{tabular}{lllll}
\hline$\alpha$ & Error $\mathbf{b}$ & Error $\mathbf{g}$ & Error $\mathbf{b}$ & Error $\mathbf{g}$ \\
\hline-1.0001 & $2.2710^{-12}$ & $2.710^{-16}$ & $2.9710^{-12}$ & $3.3310^{-16}$ \\
-1.1 & $1.510^{-11}$ & $2.510^{-16}$ & $2.1510^{-11}$ & $4.4410^{-16}$ \\
-10 & $2.0510^{-10}$ & $3.3810^{-16}$ & $2.7410^{-10}$ & $4.210^{-16}$ \\
-100 & $1.0610^{-9}$ & $3.3510^{-16}$ & $1.1610^{-9}$ & $4.4410^{-16}$ \\
$-10^{6}$ & $1.2510^{-5}$ & $3.3510^{-16}$ & $7.5510^{-6}$ & $2.2210^{-16}$ \\
\hline
\end{tabular}

Forward errors for Jacobi polynomials with $a=-1 / 3, b=1 / 7, n=60, C=10$

experiments will also show that the new algorithm do not improve significantly the accuracy when the shift has a moderate size due to the conditioning of the problem.

Let us define new variables $\left\{t_{i}\right\}_{i=1}^{n-1}$ as $t_{i}:=l_{i}+\alpha$. Then, the following new algorithm to compute the Geronimus transformation with shift $\alpha$ and parameters $C, \mu_{0}$, and $\mathscr{L}_{0}$ can be derived. Notice that the variables $l_{1}, \ldots, l_{n-1}$ have disappeared since they have been replaced by $t_{1}, \ldots, t_{n-1}$.

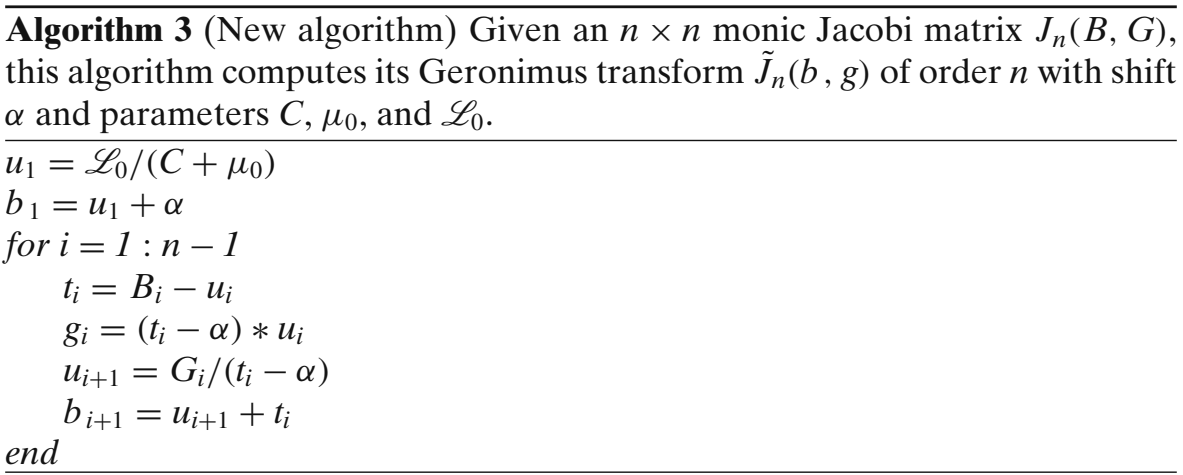

The computational cost of Algorithm 3 is $5 n-2$ flops.

A matrix version of this new algorithm is

$J_{n}(B, G)-\alpha I_{n}=U_{n}\left(T_{n}-\alpha D_{n}\right)+l_{n} e_{n} e_{n}^{t}, \quad \tilde{J}_{n}(b, g)=\left(T_{n}-\alpha D_{n}\right) U_{n}+\alpha I_{n}$,

Table 3 Algorithm 1-algorithm 2-backward algorithm

\begin{tabular}{lllllll}
\hline$\alpha$ & Error $\mathbf{b}$ & Error $\mathbf{g}$ & Error $\mathbf{b}$ & Error $\mathbf{g}$ & Error $\mathbf{b}$ & Error $\mathbf{g}$ \\
\hline-0.0001 & $2.110^{-16}$ & $3.6410^{-16}$ & $1.7210^{-15}$ & $4,3510^{-16}$ & $4.910^{-1}$ & $4.710^{-1}$ \\
-0.1 & $1.4510^{-15}$ & $2.1410^{-15}$ & $6.7610^{-15}$ & $1.0710^{-14}$ & $4.810^{-16}$ & $6.710^{-16}$ \\
-1 & $1.7110^{-6}$ & $2.8310^{-6}$ & $1.710^{-6}$ & $2.8310^{-6}$ & $710^{-7}$ & $10^{-6}$ \\
-10 & 2.74 & 44.65 & 2.74 & 44.67 & 1.4 & 2.5 \\
\hline
\end{tabular}

Forward errors for Laguerre polynomials with $a=-1 / 3, n=60, C=0$ 
Table 4 Algorithm 1-algorithm 2

\begin{tabular}{lllll}
\hline$\alpha$ & Error $\mathbf{b}$ & Error $\mathbf{g}$ & Error $\mathbf{b}$ & Error $\mathbf{g}$ \\
\hline-0.0001 & $2.0110^{-16}$ & $3.3210^{-16}$ & $1.7310^{-15}$ & $3.8610^{-16}$ \\
-0.1 & $1.0410^{-15}$ & $2.1810^{-16}$ & $1.7310^{-15}$ & $4.110^{-16}$ \\
-1 & $2.2810^{-16}$ & $2.1810^{-16}$ & $2.110^{-16}$ & $4.3610^{-16}$ \\
-10 & $3.7210^{-16}$ & $4.2610^{-16}$ & $6.1910^{-16}$ & $4.3910^{-16}$ \\
-100 & $3.9210^{-15}$ & $2.710^{-16}$ & $2.2510^{-15}$ & $2.9910^{-16}$ \\
$-10^{6}$ & $1.0810^{-10}$ & $2.1610^{-16}$ & $1.0810^{-10}$ & $4.0110^{-16}$ \\
\hline
\end{tabular}

Forward errors for Laguerre polynomials with $a=-1 / 3, n=60, C=10$

where

$U_{n}=\left(\begin{array}{ccccc}u_{1} & 1 & 0 & \ldots & 0 \\ 0 & u_{2} & 1 & \ldots & 0 \\ \vdots & \vdots & \ddots & \ddots & \vdots \\ 0 & 0 & \ldots & 0 & u_{n}\end{array}\right), \quad T_{n}=\left(\begin{array}{ccccc}1 & 0 & 0 & \ldots & 0 \\ t_{1} & 1 & 0 & \ldots & 0 \\ \vdots & \vdots & \ddots & \ddots & \vdots \\ 0 & 0 & \ldots & t_{n-1} & 1\end{array}\right), \quad D_{n}=\left(\begin{array}{ccccc}0 & 0 & 0 & \ldots & 0 \\ 1 & 0 & 0 & \ldots & 0 \\ \vdots & \vdots & \ddots & \ddots & \vdots \\ 0 & 0 & \ldots & 1 & 0\end{array}\right)$.

Some numerical results are presented in Tables 5 and 6, namely, the computed forward errors by Algorithm 3. Those tables also include the condition number, which will be defined in Subsection 4.2 and whose explicit expression is given in Theorem 3. Notice that the accuracy of the outputs increases as $|\alpha|$ increases when $C \neq 0$. However, no improvement can be observed when $C=0$.

Before carrying out a rigorous roundoff error and stability analysis of the algorithm, we can explain why the accuracy of the outputs improves when $C \neq$ 0 . Notice that the new algorithm is obtained from Algorithm 1 through some, apparently, slight modifications which actually have a significant influence on stability and accuracy.

We have observed that some harmful cancellations in the computation of the outputs $b_{i}$ by Algorithm 1 may arise. A significant situation where this problem can be clearly understood appears when the shift $\alpha$ is large. It can easily be shown that $\lim _{|\alpha| \rightarrow \infty} u_{k}=0$ for $k \geq 2$ (see Lemma 5 in Section 4.3), and therefore $l_{i}=B_{i}-\alpha-u_{i} \sim-\alpha$ when $|\alpha| \rightarrow \infty$ and $i \geq 2$, and then $b_{i+1}=$ $u_{i+1}+l_{i}+\alpha \sim(-\alpha)+\alpha$ when $|\alpha| \rightarrow \infty$ and $i \geq 2$. The reader should notice that this cancellation is avoided in Algorithm 3.

Table 5 New algorithm

\begin{tabular}{lccllll}
\hline$\alpha$ & Error b & Error $\mathbf{g}$ & cond & Error $\mathbf{b}$ & Error $\mathbf{g}$ & cond \\
\hline-1.0001 & $7.5510^{-12}$ & $2.2210^{-16}$ & $3.4610^{5}$ & $1.3410^{-12}$ & $2.710^{-16}$ & $3.5910^{4}$ \\
-1.1 & 16.04 & 1.7 & $4.8710^{16}$ & $4.0510^{-12}$ & $2.510^{-16}$ & $1.2210^{5}$ \\
-10 & 43.32 & 1.57 & $1.8310^{17}$ & $5.5310^{-13}$ & $3.3810^{-16}$ & $1.1210^{4}$ \\
-100 & 2.89 & 2.96 & $1.3310^{17}$ & $4.7410^{-14}$ & $3.3510^{-16}$ & $1.1310^{3}$ \\
-1000 & 9.69 & 10.65 & $7.6410^{16}$ & $8.410^{-15}$ & $3.3510^{-16}$ & 113.81 \\
$-10^{6}$ & 0.35 & 0.53 & $8.6110^{16}$ & $1.6410^{-15}$ & $3.3510^{-16}$ & 38.4 \\
\hline
\end{tabular}

Forward errors for Jacobi polynomials

$a=-1 / 3, b=1 / 7, n=60, C=0$ (left) and $C=10$ (right) 
Table 6 New algorithm

\begin{tabular}{llclllr}
\hline$\alpha$ & Error b & Error $\mathbf{g}$ & cond & Error $\mathbf{b}$ & Error $\mathbf{g}$ & cond \\
\hline-0.0001 & $2.1110^{-16}$ & $3.6410^{-16}$ & 4.23 & $2.1110^{-16}$ & $3.3210^{-16}$ & 4.27 \\
-0.1 & $1.4510^{-15}$ & $2.1410^{-15}$ & $1.1910^{3}$ & $1.0410^{-15}$ & $2.1810^{-16}$ & 54.64 \\
-1 & $1.7110^{-6}$ & $2.8310^{-6}$ & $2.9210^{11}$ & $2.110^{-16}$ & $2.1810^{-16}$ & 5.89 \\
-10 & 2.74 & 44.67 & $2.510^{17}$ & $1.9610^{-16}$ & $4.2610^{-16}$ & 4.48 \\
-100 & 2.24 & 3.83 & $4.9710^{17}$ & $2.1110^{-16}$ & $2.710^{-16}$ & 3 \\
$-10^{6}$ & 1.15 & 1.21 & $4.2410^{16}$ & $2.210^{-16}$ & $2.1610^{-16}$ & 3 \\
\hline
\end{tabular}

Forward errors for Laguerre polynomials

$a=-1 / 3, n=60, C=0$ (left) and $C=10$ (right)

From Lemma 5 in Section 4.3 we also observe that some harmful cancellations may occur in Algorithm 1 when $C=0$ in the computation of $b_{1}, l_{1}$, and $u_{2}$, but these are not eliminated by Algorithm 3. In fact, these cancellations cannot be eliminated because they reflect the ill-conditioning of the problem.

\subsection{Backward error analysis of Algorithm 3}

We use the standard model of floating point arithmetic [19]:

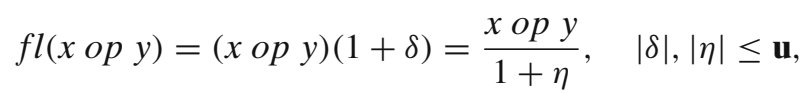

where $x$ and $y$ are floating point numbers, $o p=+,-, *, /$, and $\mathbf{u}$ is the unit roundoff of the machine. From now on, given a vector $\mathbf{v},|\mathbf{v}|$ denotes the vector whose entries are the absolute values of the entries of $\mathbf{v}$.

We develop our error analysis in the most general setting. For this purpose we assume that the shift $\alpha$ and $C$ are real numbers, and we denote by $\hat{\alpha}$ and $\hat{C}$ the nearest floating point numbers to $\alpha$ and $C$. Similarly, we denote by $\hat{\mathscr{L}}_{0}$ and $\hat{\mu}_{0}$ the nearest floating point numbers to $\mathscr{L}_{0}$ and $\mu_{0}$. Moreover, we assume that the input parameters $B_{1}, \ldots, B_{n-1}$ and $G_{1}, \ldots, G_{n-1}$ are each affected respectively by the small relative errors $\epsilon_{B_{1}}, \ldots, \epsilon_{B_{n-1}}, \epsilon_{G_{1}}, \ldots, \epsilon_{G_{n-1}}$, where $\max _{1 \leq i \leq n-1}\left\{\left|\epsilon_{B_{i}}\right|,\left|\epsilon_{G_{i}}\right|\right\} \leq D \mathbf{u}, D$ being a moderate constant. These errors in the inputs may come from the rounding process when storing them in the computer. In addition, for the Jacobi matrices associated with families of classical orthogonal polynomials, the inputs $B_{i}$ and $G_{i}$ are computed using well-known formulae which may cause further errors.

Theorem 1 Let $J_{n}(B, G)$ be a monic Jacobi matrix of order $n$. Let $\tilde{J}_{n}(b, g)$ be the Geronimus transform with shift $\alpha$ and parameters $C$, $\mu_{0}$, and $\mathscr{L}_{0}$ of $J_{n}(B, G)$. Let $\hat{\alpha}, \hat{\mu}_{0}$, and $\hat{C}$ be the nearest floating point numbers to $\alpha, \mu_{0}$, and $C$. Consider the application of Algorithm 3 to the matrix with floating point entries $J_{n}(\hat{B}, \hat{G})$ where

$$
\hat{B}_{i}=B_{i}\left(1+\epsilon_{B_{i}}\right), \quad \hat{G}_{i}=G_{i}\left(1+\epsilon_{G_{i}}\right), \quad 1 \leq i \leq n-1,
$$


and

$$
\max _{1 \leq i \leq n-1}\left\{\left|\epsilon_{B_{i}}\right|,\left|\epsilon_{G_{i}}\right|\right\} \leq D \boldsymbol{u}
$$

for a positive integer $D$ such that $D \boldsymbol{u} \ll 1$. If $\tilde{J}_{n}(\hat{b}, \hat{g})$ is the matrix computed by Algorithm 3, and $\hat{L}, \hat{T}$ are the computed intermediate matrices appearing in Algorithm 3, then

$$
\begin{gathered}
J_{n}(B+\Delta B, G+\Delta G)-\hat{\alpha} I_{n}=\hat{U}_{n}\left(\hat{T}_{n}-\hat{\alpha} I_{n}\right), \\
\tilde{J}_{n}(\hat{b}+\Delta \hat{b}, \hat{g}+\Delta \hat{g})=\left(\hat{T}_{n}-\hat{\alpha} I_{n}\right) \hat{U}_{n}+\hat{\alpha} I_{n}
\end{gathered}
$$

where this transformation has parameters $\hat{C}, \Delta \mathscr{L}_{0}$, and $\hat{\mu}_{0}$, and

$$
\begin{array}{llr}
|\hat{\alpha}-\alpha| & \leq \boldsymbol{u}|\alpha| \\
\left|\Delta \mathscr{L}_{0}\right| & \leq 3 \boldsymbol{u}\left|\mathscr{L}_{0}\right|+O\left(\boldsymbol{u}^{2}\right), & \\
\left|\hat{\mu}_{0}-\mu_{0}\right| \leq \boldsymbol{u}\left|\mu_{0}\right| & \\
|\hat{C}-C| & \leq \boldsymbol{u}|C|, \\
\left|\Delta B_{i}\right| & \leq(D+1) \boldsymbol{u}\left(\left|B_{i}\right|+\left|\hat{u}_{i}\right|\right)+O\left(\boldsymbol{u}^{2}\right), & 1 \leq i \leq n-1, \\
\left|\Delta G_{i}\right| & \leq(D+2) \boldsymbol{u}\left|G_{i}\right|+O\left(\boldsymbol{u}^{2}\right), & 1 \leq i \leq n-1, \\
\left|\Delta \hat{b}_{i}\right| & \leq \boldsymbol{u}\left|\hat{b}_{i}\right|, & 1 \leq i \leq n, \\
\left|\Delta \hat{g}_{i}\right| & \leq 2 \boldsymbol{u}\left|\hat{g}_{i}\right|+O\left(\boldsymbol{u}^{2}\right), & 1 \leq i \leq n-1 .
\end{array}
$$

Proof First observe that

$$
\hat{t}_{i}=\left(B_{i}\left(1+\epsilon_{B_{i}}\right)-\hat{u}_{i}\right)\left(1+\epsilon_{t_{i}}\right),\left|\epsilon_{t_{i}}\right| \leq \mathbf{u}
$$

and we get

$$
\left|\Delta B_{i}\right|=\left|\hat{t}_{i}+\hat{u}_{i}-B_{i}\right| \leq\left((D+1) \mathbf{u}+D \mathbf{u}^{2}\right)\left(\left|B_{i}\right|+\left|\hat{u}_{i}\right|\right) .
$$

Assume that the floating point number closer to $\mathscr{L}_{0}$ is $\mathscr{L}_{0}\left(1+\epsilon_{L}\right)$. Then,

$$
\hat{u}_{1}=\frac{\mathscr{L}_{0}\left(1+\epsilon_{L}\right)\left(1+\epsilon_{u_{1}}\right)\left(1+\delta_{u_{1}}\right)}{\hat{C}+\hat{\mu}_{0}}, \quad\left|\epsilon_{L}\right|,\left|\epsilon_{u_{1}}\right|,\left|\delta_{u_{1}}\right| \leq \mathbf{u} .
$$

Therefore,

$$
\begin{aligned}
\left|\Delta \mathscr{L}_{0}\right| & =\left|\mathscr{L}_{0}-\hat{u}_{1}\left(\hat{C}+\hat{\mu}_{0}\right)\right| \leq\left(3 \mathbf{u}+3 \mathbf{u}^{2}+\mathbf{u}^{3}\right)\left|\mathscr{L}_{0}\right| . \\
\hat{u}_{i+1} & =\frac{G_{i}\left(1+\epsilon_{G_{i}}\right)}{\hat{t}_{i}-\hat{\alpha}}\left(1+\delta_{u_{i+1}}\right)\left(1+\epsilon_{u_{i+1}}\right),\left|\delta_{u_{i+1}}\right|,\left|\epsilon_{u_{i+1}}\right| \leq \mathbf{u}
\end{aligned}
$$

which implies

$$
\left|\Delta G_{i}\right|=\left|\left(\hat{t}_{i}-\hat{\alpha}\right) \hat{u}_{i+1}-G_{i}\right| \leq\left((D+2) \mathbf{u}+(2 D+1) \mathbf{u}^{2}+D \mathbf{u}^{3}\right)\left|G_{i}\right| .
$$


Finally,

$$
\begin{gathered}
\hat{b}_{i}\left(1+\epsilon_{b_{i}}\right)=\hat{u}_{i+1}+\hat{t}_{i},\left|\epsilon_{b_{i}}\right| \leq \mathbf{u} . \\
\hat{g}_{i}\left(1+\epsilon_{g_{i}}\right)\left(1+\delta_{g_{i}}\right)=\left(\hat{t}_{i+1}-\hat{\alpha}\right) \hat{u}_{i},\left|\epsilon_{g_{i}}\right|,\left|\delta_{g_{i}}\right| \leq \mathbf{u},
\end{gathered}
$$

and the results follow in a straightforward way.

In plain words, Theorem 1 says that the computed Geronimus transform $\tilde{J}_{n}(\hat{b}, \hat{g})$ with shift $\alpha$ and parameters $C, \mu_{0}$, and $\mathscr{L}_{0}$ is almost the exact Geronimus transform of $J_{n}(B+\Delta B, G+\Delta G)$ with shift $\hat{\alpha}$ and parameters $C+\Delta C, \hat{\mu}_{0}$, and $\hat{\mathscr{L}}_{0}$.

Definition 1 [19] A method for computing $y=f(x)$ is called mixed forwardbackward stable (or numerically stable) if, for any $x$, it produces a computed $\hat{y}$ satisfying

$$
\hat{y}+\Delta \hat{y}=f(x+\Delta x), \quad|\Delta \hat{y}| \leq \epsilon|\hat{y}|, \quad|\Delta x| \leq \eta|x|,
$$

provided that $\epsilon$ and $\eta$ are sufficiently small. Informally, a mixed forwardbackward stable algorithm produces almost the right answer for almost the right data.

We conclude that Algorithm 3 is componentwise stable in a mixed forwardbackward sense [19] if $\left|\hat{u}_{i}\right|=O\left(\left|B_{i}\right|\right)$, for $1 \leq i \leq n$. However the following problem arises: $\left|\Delta B_{i}\right| /\left|B_{i}\right|$ can be much larger than $\mathbf{u}$ if $\left|\hat{u}_{i}\right|$ is much larger than $\left|B_{i}\right|$. Unfortunately, this can happen as the following numerical experiments show. Consider the sequence of Jacobi polynomials with parameters $-1 / 3,1 / 7$, and the shift $\alpha=-2$. Taking into account Theorem 1 , we compute a bound for the backward error as $(\epsilon \cdot$ errback $)$, where errback $=$ $\max _{i=1: n-1}\left\{1+\left|\hat{u}_{i} / B_{i}\right|\right\}$, and we get

\begin{tabular}{llll}
\hline & $n=10$ & $n=100$ & $n=1000$ \\
\hline errback, $C=0$ & $7.2310^{3}$ & $3.510^{5}$ & $5.910^{6}$ \\
errback, $C=10$ & 418 & $5.710^{4}$ & $5.910^{6}$ \\
\hline
\end{tabular}

The previous table shows that the upper bound of the backward error is not "small". Therefore, we cannot assure mixed forward-backward stability.

\subsection{Condition number}

The main goal of this section is to develop a bound that allows us to estimate the forward errors of Algorithm 3 in $O(n)$ operations. We also present some numerical experiments showing that the bound obtained gives a good prediction of the forward errors produced by this algorithm. 
To bound the errors in Algorithm 3, we study the sensitivity of the Geronimus transformation with respect to perturbations of the initial data, i.e., the parameters of the monic Jacobi matrix $J_{n}(B, G)$, the shift $\alpha$, and the parameters $C, \mu_{0}$ and $\mathscr{L}_{0}$. We consider perturbations associated with the backward errors found in Theorem 1 and we measure the sensitivity of the problem by using the notion of componentwise relative condition number. This condition number, together with Theorem 1, allows us to get a tight upper bound on the forward errors obtained by the application of Algorithm 3 to a monic Jacobi matrix. This bound is presented in Theorem 2. In the following definition the variables $u_{1}, u_{2}, \ldots, u_{n}$ correspond to the diagonal entries of $U$ in the UL factorization of $J_{n}(B, G)-\alpha I$.

Definition 2 Let $\tilde{J}_{n}(b, g)$ be the Geronimus transform of order $n$ with shift $\alpha$ and parameters $C, \mu_{0}$, and $\mathscr{L}_{0}$ of the $n \times n$ monic Jacobi matrix $J_{n}(B, G)$. Let $\tilde{J}_{n}(b+\Delta b, g+\Delta g)$ be the Geronimus transform of order $n$ with shift $\alpha+\Delta \alpha$ and parameters $C+\Delta C, \mu_{0}+\Delta \mu_{0}$, and $\mathscr{L}_{0}+\Delta \mathscr{L}_{0}$ of the $n \times n$ monic Jacobi matrix $J_{n}(B+\Delta B, G+\Delta G)$. Let us define

$$
D B:=\max \left\{\max _{1 \leq i \leq n-1}\left\{\frac{\left|\Delta B_{i}\right|}{\left|B_{i}\right|+\left|u_{i}\right|}\right\}, \max _{1 \leq i \leq(n-1)}\left\{\frac{\left|\Delta G_{i}\right|}{\left|G_{i}\right|}\right\}, \frac{|\Delta \alpha|}{|\alpha|}, \frac{|\Delta C|}{|C|}, \frac{\left|\Delta \mu_{0}\right|}{\left|\mu_{0}\right|}, \frac{\left|\Delta \mathscr{L}_{0}\right|}{\left|\mathscr{L}_{0}\right|},\right\},
$$

where the quotient $|\Delta \alpha| /|\alpha|$ has to be understood as zero if $\alpha=0$. Then, the relative componentwise condition number of the Geronimus transformation with shift $\alpha$ and parameters $C, \mu_{0}$, and $\mathscr{L}_{0}$ with respect to perturbations associated with the backward errors in Theorem 1 is defined as

$$
\kappa\left(B, G, \alpha, C, \mu_{0}, \mathscr{L}_{0}\right):=\lim _{\delta \rightarrow 0} \sup _{0 \leq D B \leq \delta} \frac{\max \left\{\max _{1 \leq i \leq n}\left\{\frac{\left|\Delta b_{i}\right|}{\left|b_{i}\right|}\right\}, \max _{1 \leq i \leq n-1)}\left\{\frac{\left|\Delta g_{i}\right|}{\left|g_{i}\right|}\right\}\right\}}{D B} .
$$

The condition number $\kappa\left(B, G, \alpha, C, \mu_{0}, \mathscr{L}_{0}\right)$ is infinite if some of the denominators appearing in the relative changes of the outputs $b_{i}$, i.e. $\left|\Delta b_{i}\right| /\left|b_{i}\right|$, are zero. However, $b_{i}=0$ will only happen for extremely particular values of the shift $\alpha$ and the rest of the parameters. In those cases, other condition numbers have to be considered. For instance, measuring absolute changes in the corresponding components of $b$, or measuring relative normwise changes of $b$. We do not consider these particular situations in this work. Notice that $g_{i} \neq 0$ for all $i$ since $g_{i}=\left(t_{i}-\alpha\right) u_{i}$ and both factors $l_{i}=t_{i}-\alpha$ and $u_{i}$ are nonzero.

The condition number $\kappa\left(B, G, \alpha, C, \mu_{0}, \mathscr{L}_{0}\right)$ allows us to give an upper bound on the forward errors produced by Algorithm 3, as the following theorem shows. 
Theorem 2 Let $\tilde{J}_{n}(b, g)$ and $\hat{\tilde{J}}_{n}(\hat{b}, \hat{g})$ be the exact and computed Geronimus transform with shift $\alpha$ and parameters $C, \mu_{0}$, and $\mathscr{L}_{0}$ from Algorithm 3. Then, $\max _{k}\left\{\left|\frac{b_{k}-\hat{b}_{k}}{b_{k}}\right|,\left|\frac{g_{k}-\hat{g}_{k}}{g_{k}}\right|\right\} \leq\left(\frac{(D+2) \mathbf{u}}{1-(D+2) \mathbf{u}}\right)\left(1+\kappa\left(B, G, \alpha, C, \mu_{0}, \mathscr{L}_{0}\right)\right)+O\left(\mathbf{u}^{2}\right)$,

where the left hand side of the previous inequality is a shorthand expression for (11) and D is the constant used in Theorem 1.

Proof By definition of $\kappa\left(B, G, \alpha, C, \mu_{0}, \mathscr{L}_{0}\right)$,

$$
\left|\frac{\Delta b_{i}}{b_{i}}\right| \leq \kappa\left(B, G, \alpha, C, \mu_{0}, \mathscr{L}_{0}\right) D B
$$

where

$$
\left|\frac{\Delta b_{i}}{b_{i}}\right|=\left|\frac{\hat{b}_{i}+\Delta \hat{b}_{i}-b_{i}}{b_{i}}\right|
$$

by Theorem 1 . Because of Theorem 1 again,

$$
D B \leq(D+2) \mathrm{u}+O\left(\mathrm{u}^{2}\right) .
$$

Therefore, to first order,

$$
\left|\frac{\Delta b_{i}}{b_{i}}\right|=\left|\frac{\hat{b}_{i}+\Delta \hat{b}_{i}-b_{i}}{b_{i}}\right| \leq \kappa\left(B, G, \alpha, C, \mu_{0}, \mathscr{L}_{0}\right)(D+2) \mathbf{u} .
$$

Since

$$
\left|\frac{\hat{b}_{i}-b_{i}}{b_{i}}\right|-\left|\frac{\Delta \hat{b}_{i}}{b_{i}}\right| \leq\left|\frac{\hat{b}_{i}+\Delta \hat{b}_{i}-b_{i}}{b_{i}}\right|
$$

we get

$$
\left|\frac{\hat{b}_{i}-b_{i}}{b_{i}}\right| \leq \kappa\left(B, G, \alpha, C, \mu_{0}, \mathscr{L}_{0}\right)(D+2) \mathbf{u}+\left|\frac{\Delta \hat{b}_{i}}{b_{i}}\right| .
$$

Notice that by Theorem 1 ,

$$
\left|\frac{\Delta \hat{b}_{i}}{b_{i}}\right|=\left|\frac{\Delta \hat{b}_{i}}{\hat{b}_{i}}\right|\left|\frac{\hat{b}_{i}}{b_{i}}\right| \leq \mathbf{u}\left|\frac{\hat{b_{i}}-b_{i}+b_{i}}{b_{i}}\right| \leq \mathbf{u}\left(1+\left|\frac{\hat{b_{i}}-b_{i}}{b_{i}}\right|\right) .
$$

Therefore,

$$
\left|\frac{\hat{b}_{i}-b_{i}}{b_{i}}\right| \leq(D+2) \mathrm{u}\left(1+\kappa\left(B, G, \alpha, C, \mu_{0}, \mathscr{L}_{0}\right)+\left|\frac{\hat{b}_{i}-b_{i}}{b_{i}}\right|\right)
$$

and the result follows for the $b_{i}^{\prime} s$. The result for $g_{i}$ can be proven similarly. 
We will provide a way to compute $\kappa\left(B, G, \alpha, C, \mu_{0}, \mathscr{L}_{0}\right)$, and therefore a bound on the forward errors, with $\mathcal{O}(n)$ cost. It is essential to remark that we have checked on the reliability of the bound on the forward errors running many numerical experiments, where we have observed that the bound does not overestimate significantly the actual errors. For an example, check Tables 5 and 6.

The entries $b$ and $g$ of the Geronimus transform $\tilde{J}_{n}(b, g)$ of $J_{n}(B, G)$ are rational functions of the inputs $B, G, \alpha, C, \mu_{0}$, and $\mathscr{L}_{0}$, and, as a consequence, $b$ and $g$ are differentiable functions of these parameters whenever the denominators are different from zero. Therefore, $\kappa\left(B, G, \alpha, C, \mu_{0}, \mathscr{L}_{0}\right)$ can be expressed in terms of partial derivatives [3]. More precisely:

$$
\kappa\left(B, G, \alpha, C, \mu_{0}, \mathscr{L}_{0}\right)=\max \left\{\max _{1 \leq k \leq n}\left\{\kappa\left(b_{k}\right)\right\}, \max _{1 \leq k \leq n-1}\left\{\kappa\left(g_{k}\right)\right\}\right\},
$$

where

$$
\begin{aligned}
\kappa\left(b_{k}\right) & :=\sum_{i=1}^{k-1} \kappa_{B_{i}}\left(b_{k}\right)+\sum_{i=1}^{k-1} \kappa_{G_{i}}\left(b_{k}\right)+\kappa_{\alpha}\left(b_{k}\right)+\kappa_{C}\left(b_{k}\right)+\kappa_{\mathscr{L}_{0}}\left(b_{k}\right)+\kappa_{\mu_{0}}\left(b_{k}\right), \\
\kappa\left(g_{k}\right) & :=\sum_{i=1}^{k} \kappa_{B_{i}}\left(g_{k}\right)+\sum_{i=1}^{k-1} \kappa_{G_{i}}\left(g_{k}\right)+\kappa_{\alpha}\left(g_{k}\right)+\kappa_{C}\left(g_{k}\right)+\kappa \mathscr{L}_{0}\left(g_{k}\right)+\kappa_{\mu_{0}}\left(g_{k}\right),
\end{aligned}
$$

where, for $k=1$, the sums $\sum_{i=1}^{0}$ are understood to be zero and

$$
\begin{gathered}
\kappa_{B_{i}}\left(b_{k}\right):=\left|\frac{\left|B_{i}\right|+\left|u_{i}\right|}{b_{k}} \frac{\partial b_{k}}{\partial B_{i}}\right|, \quad \kappa_{C}\left(b_{k}\right):=\left|\frac{C}{b_{k}} \frac{\partial b_{k}}{\partial C}\right|, \\
\kappa_{\alpha}\left(b_{k}\right):=\left|\frac{\alpha}{b_{k}} \frac{\partial b_{k}}{\partial \alpha}\right|, \quad \kappa_{G_{i}}\left(b_{k}\right):=\left|\frac{G_{i}}{b_{k}} \frac{\partial b_{k}}{\partial G_{i}}\right|, \\
\kappa_{\mathscr{L}_{0}}\left(b_{k}\right):=\left|\frac{\mathscr{L}_{0}}{b_{k}} \frac{\partial u_{k}}{\partial \mathscr{L}_{0}}\right|, \quad \kappa_{\mu_{0}}\left(b_{k}\right):=\left|\frac{\mu_{0}}{b_{k}} \frac{\partial b_{k}}{\partial \mu_{0}}\right|,
\end{gathered}
$$

and analogously for $\kappa\left(g_{k}\right)$.

In Theorem 3, we give recurrence relations for computing $\kappa\left(b_{k}\right)$ and $\kappa\left(g_{k}\right)$ that lead to an explicit expression for $\kappa\left(B, G, \alpha, C, \mu_{0}, \mathscr{L}_{0}\right)$. Our first step to prove Theorem 3 is to express the intermediate variables $u_{k}$ in Algorithm 3, and the outputs $b_{k}$ and $g_{k}$ as functions of the data $B, G, \alpha, C, \mu_{0}$, and $\mathscr{L}_{0}$. Then, we obtain expressions for the partial derivatives of each of these functions with respect to their arguments. A detailed proof of this theorem can be found in Appendix 1.

Theorem 3 Let $J_{n}(B, G)$ be any $n \times n$ Jacobi matrix, and let $\alpha, C, \mu_{0}$, and $\mathscr{L}_{0}$ be real numbers such that $J_{n}(B, G)-\alpha I$ has an $U L$ factorization, where 
$u_{1}=\mathscr{L}_{0} /\left(C+\mu_{0}\right)$. Let $U$ be the upper bidiagonal factor in the $U L$ factorization of $J_{n}(B, G)-\alpha I$. If $u_{1}, u_{2}, \ldots, u_{n}$ are the entries of $U$ in positions $(1,1),(2,2), \ldots,(n, n)$, then

$$
\begin{aligned}
\kappa\left(b_{1}\right)= & \left|\frac{\alpha}{b_{1}}\right|\left|1+\frac{\partial u_{1}}{\partial \alpha}\right|+\left|\frac{u_{1}}{b_{1}}\right|\left|\kappa^{*}\left(u_{1}\right)\right|, \\
\kappa\left(b_{k}\right)= & \frac{\left|u_{k}\right|}{\left|b_{k}\right|}+\frac{\left|\gamma_{k-1} u_{k}-1\right|}{\left|b_{k}\right|}\left[\left|B_{k-1}\right|+\left|u_{k-1}\right|\left(1+\kappa^{*}\left(u_{k-1}\right)\right)\right] \\
& +\frac{|\alpha|}{\left|b_{k}\right|}\left|\left(\gamma_{k-1} u_{k}-1\right) \frac{\partial u_{k-1}}{\partial \alpha}+\gamma_{k-1} u_{k}\right|, \quad k \geq 2, \\
\kappa\left(g_{k}\right)= & \left|\gamma_{k}\right|\left[\left|B_{k}\right|+\left|u_{k}\right|+\left|\delta_{k}\right| \kappa^{*}\left(u_{k}\right)\right]+\left|\frac{\alpha}{g_{k}}\right|\left|\delta_{k} \frac{\partial u_{k}}{\partial \alpha}-u_{k}\right|, \quad k \geq 1,
\end{aligned}
$$

where

$$
\begin{gathered}
\kappa^{*}\left(u_{1}\right)=1+\frac{|C|+\left|\mu_{0}\right|}{\left|C+\mu_{0}\right|}, \\
\kappa^{*}\left(u_{k}\right)=1+\left|\gamma_{k-1}\right|\left[\left|B_{k-1}\right|+\left|u_{k-1}\right|\left(1+\kappa^{*}\left(u_{k-1}\right)\right], \quad k \geq 2,\right. \\
\frac{\partial u_{k}}{\partial \alpha}= \begin{cases}-\frac{u_{1}}{C+\mu_{0}} \frac{\partial \mu_{0}}{\partial \alpha}, & k=1 \\
\gamma_{k-1} u_{k}\left(1+\frac{\partial u_{k-1}}{\partial \alpha}\right), & k>1\end{cases}
\end{gathered}
$$

and

$$
\gamma_{k}:=\frac{1}{B_{k}-u_{k}-\alpha}, \quad \delta_{k}:=B_{k}-2 u_{k}-\alpha, \quad k \geq 1 .
$$

Remark 1 It is possible to develop a roundoff error analysis of Algorithm 1 similar to the analysis done for Algorithm 3. To begin with, backward error bounds for Algorithm 1 can be found. Then, it is also possible to deduce recurrence relations for a relative componentwise condition number, $\kappa_{A}\left(B, G, \alpha, C, \mu_{0}, \mathscr{L}_{0}\right)$, for the Geronimus transformation with respect to perturbations in the input data associated with the backward errors of Algorithm 1. Finally, the condition number $\kappa_{A}\left(B, G, \alpha, C, \mu_{0}, \mathscr{L}_{0}\right)$ can be used in a counterpart version of Theorem 2 for Algorithm 1 to bound the forward errors. We do not include the details of these results to keep the paper concise. However, we would like to remark that it is easy to prove that

$$
\kappa\left(B, G, \alpha, C, \mu_{0}, \mathscr{L}_{0}\right) \leq \kappa_{A}\left(B, G, \alpha, C, \mu_{0}, \mathscr{L}_{0}\right)
$$

for all monic Jacobi matrices $J_{n}(B, G)$, all shifts $\alpha$, and all the possible values of the parameters $C, \mu_{0}$ and $\mathscr{L}_{0}$. This fact, together with the numerical experiments in Subsection 3.1, show that Algorithm 3 is more accurate than Algorithm 1.

Similar remarks can be made regarding Algorithm 2. 
4.3 Stability and accuracy of the new algorithm for large shifts

There are some interesting results that we can prove related to the stability and accuracy of Algorithm 3 beyond the fact of being more accurate than Algorithm 1 or 2 . It can be proven that, for large enough values of the shift $\alpha$ and under some small constraints, for $C \neq 0$, Algorithm 3 is accurate, i.e., it produces outputs with componentwise forward errors of order $O(\mathbf{u})$. To prove this, we will show that

$$
\lim _{|\alpha| \rightarrow \infty} \kappa\left(B, G, \alpha, C, \mu_{0}, \mathscr{L}_{0}\right)=\max \left\{3, \frac{\left|B_{1}\right|+3\left|\mathscr{L}_{0} / C\right|}{\left|B_{1}-\mathscr{L}_{0} / C\right|}\right\}
$$

Therefore, Theorem 2 guarantees accuracy if the quantity on the right is "small". The numerical experiments in Subsection 3.1 show that this is not the case for Algorithms 1,2, or the backward algorithm. In fact, it can be proven that the accuracy of those algorithms decreases as $|\alpha|$ grows.

Let us recall that, if $C \neq 0$, according to Theorem 1 , if $\left|\hat{u}_{i}\right|=O\left(\left|B_{i}\right|\right)$ for $1 \leq i \leq n$, then Algorithm 3 is mixed forward-backward stable, which is the usual requirement for a numerical algorithm to be considered stable [19, p. 7]. More precisely, in this case, it can be said that the computed Geronimus transform $\tilde{J}_{n}(\hat{b}, \hat{g})$ with shift $\alpha$ and parameters $C, \mu_{0}$, and $\mathscr{L}_{0}$ of $J_{n}(B, G)$ is an $O(\mathbf{u})$ relative componentwise perturbation of the exact Geronimus transform with shift $\hat{\alpha}$ and parameters $\hat{C}, \hat{\mu}_{0}$, and $\Delta \mathscr{L}_{0}$ of $J_{n}(B+\Delta B, G+\Delta G)$, where $\Delta \mathscr{L}_{0}, \Delta B$ and $\Delta G$ are $O(\mathbf{u})$ relative componentwise perturbations of the exact inputs $\mathscr{L}_{0}, B$ and $G$. In this context, another goal of this subsection is to prove that for large enough values of the shift, $\left|u_{i}\right| \ll\left|B_{i}\right|$ and then Algorithm 3 is stable. We have to admit that this will be proven for the exact values of $u_{i}$ and not for the computed values $\hat{u}_{i}$, thus we are only proving stability up to $O\left(\mathbf{u}^{2}\right)$ terms.

Here we will also show that the condition number $\kappa\left(B, G, \alpha, C, \mu_{0}, \mathscr{L}_{0}\right)$ becomes very large as $|\alpha|$ grows when $C=0$. In Appendix 2 we show that this condition number has the same magnitude as the standard condition number of the problem which implies that no accuracy can be expected from any algorithm to compute the Geronimus transformation when $C=0$ and the shift moves away from the support of the measure. Moreover, Lemma 5 shows that $\left|B_{1}\right|+\left|u_{1}\right| \gg\left|B_{1}\right|$ when $|\alpha|$ grows which implies that no stability can either be expected from Algorithm 3.

We start with some technical lemmas. Firstly, the different numerical behavior of the Geronimus transformation when $C=0$ and when $C \neq 0$ can be partially explained by using the following result. Notice that the parameter $u_{1}$ can be seen as a function of $\alpha$.

Lemma 5 Let $J_{n}(B, G)$ be the leading principal submatrix of a monic Jacobi matrix $J$. Let $\alpha, C, \mu_{0}$, and $\mathscr{L}_{0}$ be real numbers such that there is a unique $U L$ 
factorization of $J_{n}(B, G)-\alpha I$. Let $u_{k}, 1 \leq k \leq n$, be the main diagonal elements in the $U$ factor. Then,

$-\quad$ if $C \neq 0$,

$$
\lim _{|\alpha| \rightarrow \infty} u_{1}=\frac{\mathscr{L}_{0}}{C}, \quad \lim _{|\alpha| \rightarrow \infty} u_{k}=0, \quad k \geq 2 .
$$

As a consequence, when $C \neq 0$, Algorithm 3 is stable for $|\alpha|$ large enough if $\left|\mathscr{L}_{0} / C\right|=O\left(\left|B_{1}\right|\right)$.

$-\quad$ if $C=0$,

$$
\begin{gathered}
\lim _{|\alpha| \rightarrow \infty}\left|u_{1}\right|=\infty, \quad\left(u_{1} \sim-\alpha\right), \\
\lim _{|\alpha| \rightarrow \infty} u_{2}=\frac{G_{1}}{B_{1}}, \quad \lim _{|\alpha| \rightarrow \infty} u_{k}=0, \quad k \geq 3 .
\end{gathered}
$$

Proof First, assume $C \neq 0$. The proof follows directly from the expressions:

$$
u_{1}=\frac{\mathscr{L}_{0}}{C+\mu_{0}}, \quad u_{k}=\frac{G_{k-1}}{B_{k-1}-u_{k-1}-\alpha}, \quad k \geq 2,
$$

using induction and the fact that $\mu_{0} \rightarrow 0$, since

$$
\lim _{|\alpha| \rightarrow \infty} \alpha \mu_{0}=\lim _{|\alpha| \rightarrow \infty} \alpha \int \frac{d \mu}{x-\alpha}=-\mathscr{L}_{0} .
$$

The limit and the integral can be interchanged if $\alpha \notin$ supp $\mu$, because $\alpha /(x-$ $\alpha)$ is a continuous function. As a consequence, $u_{1} \rightarrow \mathscr{L}_{0} / C$ when $|\alpha| \rightarrow \infty$ if $C \neq 0$.

When $C=0$, the previous result gives $u_{1} \sim-\alpha$, so $u_{2} \rightarrow G_{1} / B_{1}$ when $|\alpha| \rightarrow$ $\infty$. This implies that $u_{3} \rightarrow 0$ and the second claim follows by induction.

Lemma 6 When $C \neq 0$ it is true that $\gamma_{k} \rightarrow 0$ and $\gamma_{k} \delta_{k} \rightarrow 1$ when $|\alpha| \rightarrow \infty$, $k \geq 1$.

Proof It follows from the definition of $\gamma_{k}$ and $\delta_{k}$ and the asymptotic properties of $u_{k}$ in Lemma 5.

Lemma 7 Let $J_{n}(B, G)$ be the leading principal submatrix of a monic Jacobi matrix $J$ of size $n$. Let $\alpha, C, \mu_{0}$, and $\mathscr{L}_{0}$ be real numbers such that there is a unique $U L$ factorization of $J_{n}(B, G)-\alpha I$. Let $u_{1}$ be the element in position $(1,1)$ in the $U$ factor. Then,

$$
\lim _{|\alpha| \rightarrow \infty} \frac{\partial u_{k}}{\partial \alpha}=\left\{\begin{aligned}
-1, & C=0 \text { and } k=1, \\
0, & \text { otherwise. }
\end{aligned}\right.
$$

Proof Taking into account the definition of $u_{1}$, when $\alpha \notin \operatorname{supp} \mu$ then

$$
\frac{\partial u_{1}}{\partial \alpha}=\frac{-\mathscr{L}_{0}}{\left(C+\mu_{0}\right)^{2}} \frac{\partial \mu_{0}}{\partial \alpha}=-\frac{\mathscr{L}_{0}}{\left(C+\mu_{0}\right)^{2}} \int_{a}^{b} \frac{d \mu}{(x-\alpha)^{2}} .
$$


The result follows from the observation that

$$
\mu_{0}=-\frac{\mathscr{L}_{0}}{\alpha}+\mathcal{O}\left(\alpha^{-2}\right), \quad \frac{\partial \mu_{0}}{\partial \alpha}=\frac{\mathscr{L}_{0}}{\alpha^{2}}+\mathcal{O}\left(\alpha^{-3}\right), \quad|\alpha| \rightarrow \infty .
$$

For $k \geq 2$ we can use induction on $k$, noting that

$$
\frac{\partial u_{k}}{\partial \alpha}=\frac{u_{k}}{B_{k-1}-u_{k-1}-\alpha}\left(1+\frac{\partial u_{k-1}}{\partial \alpha}\right) .
$$

and considering Lemma 5. Note that $\partial u_{k} / \partial \alpha=\mathcal{O}\left(\alpha^{-1}\right)$ when $|\alpha| \rightarrow \infty$.

4.4 Asymptotic analysis of the condition number when $C \neq 0$

In this subsection we present an analysis of the condition number of Algorithm 3 when $C \neq 0$.

Lemma 8 If $C \neq 0$, then

$$
\lim _{|\alpha| \rightarrow \infty} \frac{B_{1}-2 u_{1}-\alpha}{u_{1}} \frac{\partial u_{1}}{\partial \alpha}=0, \quad \lim _{|\alpha| \rightarrow \infty} \alpha \frac{\partial u_{k}}{\partial \alpha}=0, \quad k \geq 1
$$

Proof From the previous estimations it follows that when $C \neq 0$ then

$$
\frac{\partial u_{1}}{\partial \alpha}=-\frac{\mathscr{L}_{0}^{2}}{C \alpha^{2}}+\mathcal{O}\left(\alpha^{-3}\right)
$$

so the second part of the lemma is true for $k=1$. Assume that the result holds for $k-1$. Then, notice that

$$
\alpha \frac{\partial u_{k}}{\partial \alpha}=u_{k} \frac{\alpha}{B_{k-1}-u_{k-1}-\alpha}+\frac{u_{k}}{B_{k-1}-u_{k-1}-\alpha}\left(\alpha \frac{\partial u_{k-1}}{\partial \alpha}\right) .
$$

Taking limits the second result follows. The first part of the lemma is obtained directly from the asymptotic estimations of $\partial u_{1} / \partial \alpha$ and $\alpha \partial u_{1} / \partial \alpha$ given above and the fact that $u_{1} \rightarrow \mathscr{L}_{0} / C$ when $\alpha \rightarrow \infty$ and $C \neq 0$.

Theorem 4 If $C \neq 0$, then

$$
\lim _{|\alpha| \rightarrow \infty} \kappa^{*}\left(u_{1}\right)=2, \quad \lim _{|\alpha| \rightarrow \infty} \kappa^{*}\left(u_{k}\right)=1, \quad k \geq 2 .
$$

$$
\begin{gathered}
\lim _{|\alpha| \rightarrow \infty} \kappa\left(b_{k}\right)=1, \quad \text { for } k \neq 2, \quad \lim _{|\alpha| \rightarrow \infty} \kappa\left(b_{2}\right)=\frac{\left|B_{1}\right|+3\left|\mathscr{L}_{0} / C\right|}{\left|B_{1}-\mathscr{L}_{0} / C\right|} . \\
\lim _{|\alpha| \rightarrow \infty} \kappa\left(g_{1}\right)=3, \quad \lim _{|\alpha| \rightarrow \infty} \kappa\left(g_{k}\right)=1, \quad k \geq 2 .
\end{gathered}
$$


Proof We prove the result by induction on $k$. Since $\lim _{|\alpha| \rightarrow \infty} \mu_{0}=0$,

$$
\lim _{|\alpha| \rightarrow \infty} \kappa^{*}\left(u_{1}\right)=2 .
$$

It is easy to show that $\kappa^{*}\left(u_{2}\right)=1$. Assume that $\lim _{|\alpha| \rightarrow \infty} \kappa^{*}\left(u_{k-1}\right)=1$ for some $k \geq 3$. Then, taking into account Lemma 5, we get

$$
\lim _{|\alpha| \rightarrow \infty}\left|\gamma_{k-1} B_{k-1}\right|=0, \quad \lim _{|\alpha| \rightarrow \infty}\left|\gamma_{k-1} u_{k-1}\right|=0,
$$

which implies the result for $u_{k}$. Recall that $b_{1}=u_{1}+\alpha$. Then, taking into account Theorem 4, Lemmas 5 and 7 the result follows for $k=1$. For $k=2$, we apply Theorem 4, Lemmas 5-8, bearing in mind that $b_{k}=B_{k-1}+u_{k}-u_{k-1}$, $k \geq 2$.

$$
\begin{gathered}
\lim _{|\alpha| \rightarrow \infty} \frac{\left|u_{2}\right|}{\left|b_{2}\right|}=0, \quad \lim _{|\alpha| \rightarrow \infty} \frac{\left|B_{1}\right|+\left|u_{1}\right|}{\left|b_{2}\right|}=\frac{\left|B_{1}\right|+\left|\mathscr{L}_{0} / C\right|}{\left|B_{1}-\mathscr{L}_{0} / C\right|}, \\
\lim _{|\alpha| \rightarrow \infty}\left|\gamma_{1} u_{2}-1\right|\left|\frac{u_{1}}{b_{2}}\right| \kappa^{*}\left(u_{1}\right)=2\left|\frac{\mathscr{L}_{0} / C}{B_{1}-\mathscr{L}_{0} / C}\right|, \\
\lim _{|\alpha| \rightarrow \infty}\left|\frac{\alpha}{b_{2}}\right|\left|\frac{\partial b_{2}}{\partial \alpha}\right|=\lim _{|\alpha| \rightarrow \infty}\left|\frac{\alpha}{b_{2}}\right|\left|\frac{\partial u_{2}}{\partial \alpha}-\frac{\partial u_{1}}{\partial \alpha}\right|=0 .
\end{gathered}
$$

Let $k \geq 3$, then

$$
\begin{gathered}
\lim _{|\alpha| \rightarrow \infty} \frac{\left|u_{k}\right|}{\left|b_{k}\right|}=0, \quad \lim _{|\alpha| \rightarrow \infty}\left|\gamma_{k-1} u_{k}-1\right| \frac{\left|B_{k-1}\right|+\left|u_{k-1}\right|}{\left|b_{k}\right|}=1, \\
\lim _{|\alpha| \rightarrow \infty}\left|\gamma_{k-1} u_{k}-1\right|\left|\frac{u_{k-1}}{b_{k}}\right| \kappa^{*}\left(u_{k-1}\right)=0 \\
\lim _{|\alpha| \rightarrow \infty}\left|\frac{\alpha}{b_{k}}\right|\left|\frac{\partial b_{k}}{\partial \alpha}\right|=\lim _{|\alpha| \rightarrow \infty}\left|\frac{\alpha}{b_{k}}\right|\left|\frac{\partial u_{k}}{\partial \alpha}-\frac{\partial u_{k-1}}{\partial \alpha}\right|=0
\end{gathered}
$$

by Lemma 8 and the result for $b_{k}$ follows. Finally, for $k=1$,

$$
\begin{aligned}
& \lim _{|\alpha| \rightarrow \infty}\left|\gamma_{1}\right|\left[\left|B_{1}\right|+\left|u_{1}\right|\right]=0, \quad \lim _{|\alpha| \rightarrow \infty}\left|\gamma_{1} \delta_{1}\right| \kappa^{*}\left(u_{1}\right)=2, \\
& \lim _{|\alpha| \rightarrow \infty}\left|\frac{\alpha}{g_{1}}\right|\left|\left(B_{1}-2 u_{1}-\alpha\right) \frac{\partial u_{1}}{\partial \alpha}-u_{1}\right| \\
& =\lim _{|\alpha| \rightarrow \infty}\left|\frac{\alpha}{B_{1}-u_{1}-\alpha}\right|\left|\frac{B_{1}-2 u_{1}-\alpha}{C+\mu_{0}} \frac{\partial \mu_{0}}{\partial \alpha}-1\right|=1 .
\end{aligned}
$$

The last equality follows from Lemma 8. 
For $k \geq 2$, notice that

$$
\begin{gathered}
\lim _{|\alpha| \rightarrow \infty}\left|\gamma_{k}\right|\left[\left|B_{k}\right|+\left|u_{k}\right|\right]=0, \quad \lim _{|\alpha| \rightarrow \infty}\left|\gamma_{k} \delta_{k}\right| \kappa^{*}\left(u_{1}\right)=1, \\
\lim _{|\alpha| \rightarrow \infty}\left|\frac{\alpha}{g_{k}}\right|\left|\left(B_{k}-2 u_{k}-\alpha\right) \frac{\partial u_{k}}{\partial \alpha}-u_{k}\right| \\
=\left|\frac{\alpha}{B_{k}-u_{k}-\alpha}\right|\left|\frac{B_{k}-2 u_{k}-\alpha}{u_{k}} \frac{u_{k}}{B_{k-1}-u_{k-1}-\alpha}\left(1+\frac{\partial u_{k-1}}{\partial \alpha}\right)-1\right|=0 .
\end{gathered}
$$

taking into account Lemma 7.

Theorem 5 Let $\kappa\left(B, G, \alpha, C, \mu_{0}, \mathscr{L}_{0}\right)$ be the condition number for the Geronimus transformation with shift $\alpha$ and parameters $C \neq 0, \mu_{0}$, and $\mathscr{L}_{0}$ introduced in Definition 12. Then

$$
\lim _{|\alpha| \rightarrow \infty} \kappa\left(B, G, \alpha, C, \mu_{0}, \mathscr{L}_{0}\right)=\max \left\{3, \frac{\left|B_{1}\right|+3\left|\mathscr{L}_{0} / C\right|}{\left|B_{1}-\mathscr{L}_{0} / C\right|}\right\} .
$$

This implies that Algorithm 3 is accurate for $|\alpha|$ large enough as long as $\frac{\left|B_{1}\right|+3\left|\mathscr{L}_{0} / C\right|}{\left|B_{1}-\mathscr{L}_{0} / C\right|}$ is small.

Proof It is a direct consequence of Theorems 3 and 4.

4.5 Asymptotic analysis of the condition number when $C=0$

Next we present a similar analysis of the condition number for the case $C=0$. Note the different behavior of $\kappa\left(b_{k}\right)$ and $\kappa\left(g_{k}\right)$ with respect to the previous subsection.

Theorem 6 If $C=0$, then

$$
\begin{gathered}
\lim _{|\alpha| \rightarrow \infty} \kappa^{*}\left(u_{1}\right)=2, \quad \lim _{|\alpha| \rightarrow \infty} \kappa^{*}\left(u_{2}\right)=\infty \\
\lim _{|\alpha| \rightarrow \infty} \kappa^{*}\left(u_{3}\right)=1+3\left|\frac{G_{1}}{B_{1}^{2}}\right|, \quad \lim _{|\alpha| \rightarrow \infty} \kappa^{*}\left(u_{k}\right)=1, \quad \text { for all } k \geq 4, \\
\lim _{|\alpha| \rightarrow \infty} \kappa\left(b_{1}\right)=\infty, \quad \lim _{|\alpha| \rightarrow \infty} \kappa\left(b_{2}\right)=3\left|1-\frac{G_{1}}{B_{1}^{2}}\right|, \\
\lim _{|\alpha| \rightarrow \infty} \kappa\left(b_{3}\right)=\infty, \quad \lim _{|\alpha| \rightarrow \infty} \kappa\left(b_{k}\right)=1, \quad \text { for } k \geq 4 \\
\lim _{|\alpha| \rightarrow \infty} \kappa\left(g_{1}\right)=\infty, \quad \lim _{|\alpha| \rightarrow \infty} \kappa\left(g_{2}\right)=\infty, \\
\lim _{|\alpha| \rightarrow \infty} \kappa\left(g_{3}\right)=1+3\left|\frac{G_{1}}{B_{1}^{2}}\right|, \quad \lim _{|\alpha| \rightarrow \infty} \kappa\left(g_{k}\right)=1, \quad \text { for } k \geq 4
\end{gathered}
$$


Proof The result for $u_{1}$ follows in a straightforward way. In the expression for $\kappa^{*}\left(u_{2}\right)$ notice that

$$
\lim _{|\alpha| \rightarrow \infty} \frac{\left|u_{1}\right|}{\left|B_{1}-u_{1}-\alpha\right|}\left(1+\kappa^{*}\left(u_{1}\right)\right)=\infty
$$

taking into account Lemma 5.

Notice that $\kappa^{*}\left(u_{3}\right)$ can also be expressed as

$$
\begin{aligned}
1+ & \frac{\left|B_{2}\right|}{\left|B_{2}-u_{2}-\alpha\right|}+\frac{\left|u_{2}\right|}{\left|B_{2}-u_{2}-\alpha\right|}\left(2+\frac{\left|B_{1}\right|}{\left|B_{1}-u_{1}-\alpha\right|}\right) \\
& +\frac{\left|u_{2}\right|}{\left|B_{1}-u_{1}-\alpha\right|} \frac{\left|u_{1}\right|}{\left|B_{2}-u_{2}-\alpha\right|}\left(1+\kappa^{*}\left(u_{1}\right)\right) .
\end{aligned}
$$

Notice that the limit when $|\alpha|$ grows of the first three terms in the previous expression is 1 , while the limit of the last term is $3\left|G_{1} / B_{1}^{2}\right|$. Now it is easy to show the result for $k=4$. The rest of the cases follow by induction.

In order to compute the condition numbers of the $b$ 's, note first that

$$
\kappa\left(b_{1}\right)=\left|\frac{\alpha}{b_{1}}\right|\left|1+\frac{\partial u_{1}}{\partial \alpha}\right|+2\left|\frac{u_{1}}{b_{1}}\right| .
$$

Taking into account Lemmas 7 and 5, the result follows. In a similar way it is possible to prove the result for the other condition numbers $\kappa\left(b_{k}\right)$ and $\kappa\left(g_{k}\right)$.

The previous results suggest that better accuracy can be obtained when computing the Geronimus transformation with $C=0$ using the new algorithm (Algorithm 3) if at least the following outputs are computed with extended accuracy: $u_{1}, u_{2}, u_{3}, b_{1}, b_{2}, b_{3}, b_{4}, g_{1}, g_{2}, g_{3}$ and then use these values as inputs of the same algorithm. Check Table 7 for new numerical results. The computations of the 4-by-4 principal leading submatrix of the Geronimus transform $\tilde{J}$ as well as the the first three main diagonal entries of the factor $U$ were done with 64 decimal digits of precision.

Table 7 Algorithm with extended accuracy

\begin{tabular}{lcllll}
\hline$\alpha$ & Error b & Error $\mathbf{g}$ & $\alpha$ & Error $\mathbf{b}$ & Error $\mathbf{g}$ \\
\hline-1.0001 & $1.3110^{-11}$ & $2.2210^{-16}$ & -0.0001 & $2.110^{-16}$ & $3.6410^{-16}$ \\
-1.1 & 91.26 & 1.74 & -0.1 & $1.8310^{-16}$ & $2.3110^{-16}$ \\
-2 & $9.310^{-3}$ & $1.6710^{-2}$ & -1 & $1.4110^{-7}$ & $2.3410^{-7}$ \\
-10 & $1.4110^{-5}$ & $5.7310^{-7}$ & -10 & $4.510^{-3}$ & $9.310^{-3}$ \\
-100 & $5.2910^{-10}$ & $5.2810^{-10}$ & -100 & $2.3810^{-8}$ & $410^{-8}$ \\
-1000 & $1.5910^{-12}$ & $1.5910^{-12}$ & -1000 & $3.6510^{-12}$ & $3.5910^{-12}$ \\
$-10^{6}$ & $2.2110^{-16}$ & $2.2210^{-16}$ & $-10^{6}$ & $2.210^{-16}$ & $2.8910^{-16}$ \\
\hline
\end{tabular}

Forward errors for $n=60$ and $C=0$. On the left, Jacobi polynomials with $a=-1 / 3, b=1 / 7$. On the right, Laguerre polynomials with $a=-1 / 3$ 


\subsection{Forward stability of Algorithm 3}

The purpose of this section is to prove that the forward error bound we have found for Algorithm 3 is the best one can expect, because it reflects the sensitivity of the transformation to componentwise relative perturbations in the data. We have seen that Algorithm 3 is neither backward stable nor stable in the mixed backward-forward sense, and therefore we consider a weaker notion of stability. An algorithm is said to be forward stable if it produces forward errors of similar magnitude to those produced by a backward stable algorithm $[19$, p. 9]. In this section we show that Algorithm 3 is componentwise forward stable. In order to prove that, we define the relative componentwise condition number of the Geronimus transformation with shift $\alpha$ and parameters $C, \mu_{0}$, and $\mathscr{L}_{0}$ with respect to small componentwise relative perturbations of $B, G, \alpha$, $C, \mu_{0}$, and $\mathscr{L}_{0}$.

$\kappa_{S}\left(B, G, \alpha, C, \mu_{0}, \mathscr{L}_{0}\right)=\lim _{\delta \rightarrow 0} \sup _{0 \leq D C \leq \delta} \frac{\max \left\{\max _{1 \leq i \leq(n)}\left\{\frac{\left|\Delta b_{i}\right|}{\left|b_{i}\right|}\right\}, \max _{1 \leq i \leq(n-2)}\left\{\frac{\left|\Delta g_{i}\right|}{\left|g_{i}\right|}\right\}\right\}}{D C}$,

where

$D C=\max \left\{\max _{1 \leq i \leq(n)}\left\{\frac{\left|\Delta B_{i}\right|}{\left|B_{i}\right|}\right\}, \max _{1 \leq i \leq(n-1)}\left\{\frac{\left|\Delta G_{i}\right|}{\left|G_{i}\right|}\right\}, \frac{|\Delta \alpha|}{|\alpha|}, \frac{|\Delta C|}{|C|}, \frac{\left|\Delta \mu_{0}\right|}{\left|\mu_{0}\right|}, \frac{\left|\Delta \mathscr{L}_{0}\right|}{\left|\mathscr{L}_{0}\right|}\right\}$.

Recurrent expressions for $\kappa_{S}\left(B, G, \alpha, C, \mu_{0}, \mathscr{L}_{0}\right)$ can be obtained in a similar way as we got recurrent expressions for $\kappa\left(B, G, \alpha, C, \mu_{0}, \mathscr{L}_{0}\right)$.

Theorem 7 Let $J_{n}(B, G)$ be any $n \times n$ monic Jacobi matrix, and let $\alpha, C, \mu_{0}$, and $\mathscr{L}_{0}$ be real numbers such that $J_{n}(B, G)-\alpha I$ has a unique $U L$ factorization with $u_{1}=\mathscr{L}_{0} /\left(C+\mu_{0}\right)$. Let $U$ be the upper bidiagonal factor in the $U L$ factorization of $J_{n}(B, G)-\alpha$ I. If $u_{1}, u_{2}, \ldots, u_{n}$ are the entries of $U$ in positions $(1,1),(2,2), \ldots,(n, n)$, then

$$
\begin{aligned}
\kappa_{S}\left(b_{1}\right)= & \left|\frac{\alpha}{b_{1}}\right|\left|1+\frac{\partial u_{1}}{\partial \alpha}\right|+\left|\frac{u_{1}}{b_{1}}\right|\left|\kappa_{S}^{*}\left(u_{1}\right)\right|, \\
\kappa_{S}\left(b_{k}\right)= & \frac{\left|u_{k}\right|}{\left|b_{k}\right|}+\frac{\left|\gamma_{k-1} u_{k}-1\right|}{\left|b_{k}\right|}\left[\left|B_{k-1}\right|+\left|u_{k-1}\right| \kappa_{S}^{*}\left(u_{k-1}\right)\right], \\
& +\left|\frac{\alpha}{b_{k}}\right|\left|\left(\gamma_{k-1} u_{k}-1\right) \frac{\partial u_{k-1}}{\partial \alpha}+\gamma_{k-1} u_{k}\right|, \quad k \geq 2, \\
\kappa_{S}\left(g_{k}\right)= & \left|\gamma_{k}\right|\left[\left|B_{k}\right|+\left|\delta_{k}\right| \kappa_{S}^{*}\left(u_{k}\right)\right]+\left|\frac{\alpha}{g_{k}}\right|\left|\delta_{k} \frac{\partial u_{k}}{\partial \alpha}-u_{k}\right|, \quad k \geq 1,
\end{aligned}
$$


where

$$
\begin{aligned}
& \kappa_{S}^{*}\left(u_{1}\right)=1+\frac{|C|+\left|\mu_{0}\right|}{\left|C+\mu_{0}\right|}, \\
& \kappa_{S}^{*}\left(u_{k}\right)=1+\left|\gamma_{k-1}\right|\left[\left|B_{k-1}\right|+\left|u_{k-1}\right| \kappa_{S}^{*}\left(u_{k-1}\right)\right], \quad k \geq 2,
\end{aligned}
$$

and

$$
\frac{\partial u_{k}}{\partial \alpha}= \begin{cases}-\frac{u_{1}}{C+\mu_{0}} \frac{\partial \mu_{0}}{\partial \alpha}, & k=1, \\ \gamma_{k-1} u_{k}\left(1+\frac{\partial u_{k-1}}{\partial \alpha}\right), & k>1 .\end{cases}
$$

Proof Analogous to the proof of Theorem 3.

To prove that Algorithm 3 is componentwise forward stable is equivalent to prove that $\kappa_{S}\left(B, G, \alpha, C, \mu_{0}, \mathscr{L}_{0}\right)$ and $\kappa\left(B, G, \alpha, C, \mu_{0}, \mathscr{L}_{0}\right)$ have the same order of magnitude, by taking into account Theorem 2.

By using Theorem 7, we can prove Theorem 8, after considerably long and delicate algebraic manipulations are performed. The complete proof can be found in Appendix 2. This theorem states that the condition numbers, $\kappa\left(B, G, \alpha, C, \mu_{0}, \mathscr{L}_{0}\right)$ and $\kappa_{S}\left(B, G, \alpha, C, \mu_{0}, \mathscr{L}_{0}\right)$ that we have defined for the Geronimus transformation are of the same order of magnitude, which implies that Algorithm 3 is forward stable.

Theorem 8 Let $\kappa\left(B, G, \alpha, C, \mu_{0}, \mathscr{L}_{0}\right)$ and $\kappa_{S}\left(B, G, \alpha, C, \mu_{0}, \mathscr{L}_{0}\right)$ be the condition numbers introduced, respectively, in Definition 2 and (18) for the Geronimus transformation with shift $\alpha$ and parameters $C, \mu_{0}$ and $\mathscr{L}_{0}$, then

$$
\kappa_{S}\left(B, G, \alpha, C, \mu_{0}, \mathscr{L}_{0}\right) \leq \kappa\left(B, G, \alpha, C, \mu_{0}, \mathscr{L}_{0}\right) \leq 8 \kappa_{S}\left(B, G, \alpha, C, \mu_{0}, \mathscr{L}_{0}\right) .
$$

This result together with the fact that $\kappa\left(B, G, \alpha, C, \mu_{0}, \mathscr{L}_{0}\right) \geq 1$ implies that Algorithm 3 is componentwise forward stable.

Acknowledgements The authors are very thankful to the two anonymous referees, whose comments were very useful to improve the presentation and content of this paper.

Open Access This article is distributed under the terms of the Creative Commons Attribution Noncommercial License which permits any noncommercial use, distribution, and reproduction in any medium, provided the original author(s) and source are credited.

\section{Appendix 1: Proof of Theorem 3}

In this section we include the proof of Theorem 3. First, we express the intermediate variables $u_{k}$ of Algorithm 1 , and the outputs $b_{k}$ and $g_{k}$ as functions of the data $B, G, \alpha, C, \mu_{0}$, and $\mathscr{L}_{0}$. Then we obtain expressions of 
the partial derivatives of each of the functions with respect to their arguments. From Algorithm 1, we get

$$
u_{1}=\frac{\mathscr{L}_{0}}{C+\mu_{0}}, \quad u_{k}=\frac{G_{k-1}}{B_{k-1}-u_{k-1}-\alpha}, \quad k \geq 2,
$$

and hence, for $k \geq 2, \quad u_{k}$ can be seen as a function of $B_{1}, \ldots, B_{k-1}, G_{1}, \ldots, G_{k-1}, \alpha, C, \mu_{0}$, and $\mathscr{L}_{0}$. Notice that $u_{1}$ is a function of $\alpha, C, \mu_{0}$, and $\mathscr{L}_{0}$ only.

The proofs of the next three lemmas have been omitted because the results can easily be obtained through direct computations.

Lemma 9 If $\alpha, C, \mu_{0}$ and $\mathscr{L}_{0}$ are real numbers such that $J_{n}(B, G)-\alpha I$ has a unique $U L$ factorization, then $u_{k}$ has the following partial derivatives with respect to $B_{1}, \ldots, B_{k-1}, G_{1}, \ldots, G_{k-1}, \alpha, C, \mu_{0}$ and $\mathscr{L}_{0}$.

$$
\begin{aligned}
& \frac{\partial u_{k}}{\partial B_{i}}= \begin{cases}0, & k=1 \\
-\gamma_{k-1} u_{k}, & i=k-1, k>1 \\
\gamma_{k-1} u_{k} \frac{\partial u_{k-1}}{\partial B_{i}}, & i<k-1, k>1\end{cases} \\
& \frac{\partial u_{k}}{\partial G_{i}}= \begin{cases}0, & k=1, \\
\gamma_{k-1}, & i=k-1, k>1 \\
\gamma_{k-1} u_{k} \frac{\partial u_{k-1}}{\partial G_{i}}, & i<k-1, k>1\end{cases} \\
& \frac{\partial u_{k}}{\partial \alpha}= \begin{cases}-\frac{u_{1}}{C+\mu_{0}} \frac{\partial \mu_{0}}{\partial \alpha}, & k=1 \\
\gamma_{k-1} u_{k}\left(1+\frac{\partial u_{k-1}}{\partial \alpha}\right), & k>1\end{cases} \\
& \frac{\partial u_{k}}{\partial C}= \begin{cases}\frac{-u_{1}}{C+\mu_{0}}, & k=1 \\
\gamma_{k-1} u_{k} \frac{\partial u_{k-1}}{\partial C}, & k>1\end{cases} \\
& \frac{\partial u_{k}}{\partial \mathscr{L}_{0}}=\left\{\begin{array}{cc}
\frac{1}{C+\mu_{0}}, & k=1 \\
\gamma_{k-1} u_{k} \frac{\partial u_{k-1}}{\partial \mathscr{L}_{0}}, & k>1
\end{array}\right. \\
& \frac{\partial u_{k}}{\partial \mu_{0}}= \begin{cases}\frac{-u_{1}}{C+\mu_{0}}, & k=1 \\
\gamma_{k-1} u_{k} \frac{\partial u_{k-1}}{\partial \mu_{0}}, & k>1\end{cases}
\end{aligned}
$$


Here

$$
\gamma_{k-1}:=\frac{1}{B_{k-1}-u_{k-1}-\alpha}, \quad k \geq 2 .
$$

From Algorithm 1, we also get

$$
b_{1}=u_{1}+\alpha, \quad b_{k}=B_{k-1}+u_{k}-u_{k-1}, \quad k \geq 2
$$

and, therefore, for $k \geq 2$, the variable $b_{k}$ can be seen as a function of $B_{1}, \ldots, B_{k-1}, G_{1}, \ldots, G_{k-1}, \alpha, C, \mu_{0}, \mathscr{L}_{0}$. Notice that $b_{1}$ is only a function of $\alpha$, $C, \mu_{0}$, and $\mathscr{L}_{0}$.

Lemma 10 If $\alpha, C, \mu_{0}$ and $\mathscr{L}_{0}$ are real numbers such that $J_{n}(B, G)-\alpha I$ has a unique $U L$ factorization, then the partial derivatives of $b_{k}$ with respect to $B_{1}, \ldots, B_{k-1}, G_{1}, \ldots, G_{k-1}, \alpha, C, \mu_{0}$, and $\mathscr{L}_{0}$ are

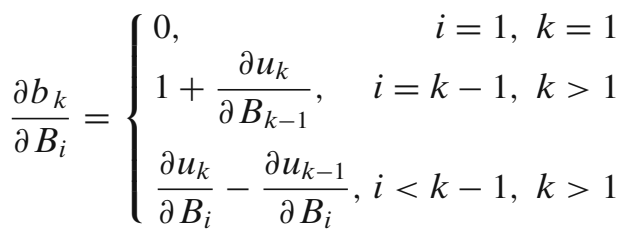

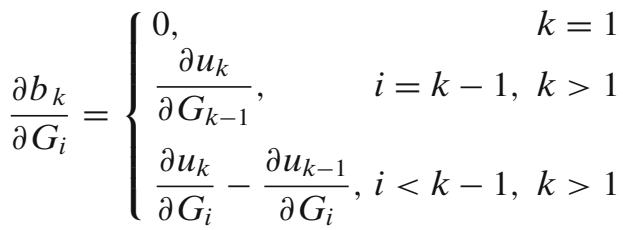

$$
\begin{aligned}
& \frac{\partial b_{k}}{\partial \alpha}= \begin{cases}\frac{\partial u_{1}}{\partial \alpha}+1, & k=1 \\
\frac{\partial u_{k}}{\partial \alpha}-\frac{\partial u_{k-1}}{\partial \alpha}, & k>1\end{cases} \\
& \frac{\partial b_{k}}{\partial C}= \begin{cases}\frac{\partial u_{1}}{\partial C}, & k=1 \\
\frac{\partial u_{k}}{\partial C}-\frac{\partial u_{k-1}}{\partial C}, & k>1\end{cases} \\
& \frac{\partial b_{k}}{\partial \mathscr{L}_{0}}= \begin{cases}\frac{\partial u_{1}}{\partial \mathscr{L}_{0}}, & k=1 \\
\frac{\partial u_{k}}{\partial \mathscr{L}_{0}}-\frac{\partial u_{k-1}}{\partial \mathscr{L}_{0}}, & k>1\end{cases} \\
& \frac{\partial b_{k}}{\partial \mu_{0}}= \begin{cases}\frac{\partial u_{1}}{\partial \mu_{0}}, & k=1 \\
\frac{\partial u_{k}}{\partial \mu_{0}}-\frac{\partial u_{k-1}}{\partial \mu_{0}}, & k>1\end{cases}
\end{aligned}
$$


It also happens that $g_{k}=\left(B_{k}-u_{k}-\alpha\right) u_{k}, k \geq 1$, so $g_{k}$ is a function of $B_{1}, \ldots, B_{k}, G_{1}, \ldots, G_{k-1}, \alpha, C, \mu_{0}, \mathscr{L}_{0}$.

Lemma 11 If $\alpha, C, \mu_{0}$ and $\mathscr{L}_{0}$ are real numbers such that $J_{n}(B, G)-\alpha I$ has a unique $U L$ factorization, then the partial derivatives of $g_{k}$ with respect to $B_{1}, \ldots, B_{k}, G_{1}, \ldots, G_{k-1}, \alpha$ and $C$ are

$$
\begin{aligned}
\frac{\partial g_{k}}{\partial B_{i}} & =\left\{\begin{array}{l}
u_{k}, \quad i=k \\
\delta_{k} \frac{\partial u_{k}}{\partial B_{i}}, i<k
\end{array}\right. \\
\frac{\partial g_{k}}{\partial G_{i}} & =\delta_{k} \frac{\partial u_{k}}{\partial G_{i}}, \quad i<k \\
\frac{\partial g_{k}}{\partial \alpha} & =\delta_{k} \frac{\partial u_{k}}{\partial \alpha}-u_{k}, \quad k \geq 1 \\
\frac{\partial g_{k}}{\partial C} & =\delta_{k} \frac{\partial u_{k}}{\partial C}, \quad k \geq 1 \\
\frac{\partial g_{k}}{\partial \mathscr{L}_{0}} & =\delta_{k} \frac{\partial u_{k}}{\partial \mathscr{L}_{0}}, \quad k \geq 1 \\
\frac{\partial g_{k}}{\partial \mu_{0}} & =\delta_{k} \frac{\partial u_{k}}{\partial \mu_{0}}, \quad k \geq 1
\end{aligned}
$$

Here $\delta_{k}:=B_{k}-2 u_{k}-\alpha$ for $k \geq 1$.

Next, we define some quantities that will be useful in order to compute the condition number $\kappa\left(B, G, \alpha, C, \mu_{0}, \mathscr{L}_{0}\right)$ introduced in (12). Let us call

$$
\kappa^{*}\left(u_{k}\right):=\sum_{i=1}^{k-1} \kappa_{B_{i}}\left(u_{k}\right)+\sum_{i=1}^{k-1} \kappa_{G_{i}}\left(u_{k}\right)+\kappa_{C}\left(u_{k}\right)+\kappa \mathscr{L}_{0}\left(u_{k}\right)+\kappa_{\mu_{0}}\left(u_{k}\right),
$$

where

$$
\begin{gathered}
\kappa_{B_{i}}\left(u_{k}\right):=\left|\frac{\left|B_{i}\right|+\left|u_{i}\right|}{u_{k}} \frac{\partial u_{k}}{\partial B_{i}}\right|, \quad \kappa_{C}\left(u_{k}\right):=\left|\frac{C}{u_{k}} \frac{\partial u_{k}}{\partial C}\right|, \\
\kappa_{G_{i}}\left(u_{k}\right):=\left|\frac{G_{i}}{u_{k}} \frac{\partial u_{k}}{\partial G_{i}}\right|, \quad \kappa_{\mathscr{L}_{0}}\left(u_{k}\right):=\left|\frac{\mathscr{L}_{0}}{u_{k}} \frac{\partial u_{k}}{\partial \mathscr{L}_{0}}\right|, \quad \kappa_{\mu_{0}}\left(u_{k}\right):=\left|\frac{\mu_{0}}{u_{k}} \frac{\partial u_{k}}{\partial \mu_{0}}\right| .
\end{gathered}
$$

Note that the subscript of these auxiliary "condition numbers" indicates with respect to which input variable the specific condition number is computed. 
The quantities $\kappa^{*}\left(u_{k}\right)$ can be computed recursively as the following lemma shows:

Lemma 12 Let $\alpha, C, \mathscr{L}_{0}$, and $\mu_{0}$ be real numbers such that $J_{n}(B, G)-\alpha I$ has a unique $U L$ factorization. Then,

$$
\begin{aligned}
\kappa^{*}\left(u_{1}\right)= & 1+\frac{|C|+\left|\mu_{0}\right|}{\left|C+\mu_{0}\right|} \\
\kappa^{*}\left(u_{k}\right)= & 1+\left|\gamma_{k-1} B_{k-1}\right|+\left|\gamma_{k-1} u_{k-1}\right|\left(1+\kappa^{*}\left(u_{k-1}\right)\right), \quad k \geq 2, \\
\kappa\left(b_{1}\right)= & \left|\frac{\alpha}{u_{1}+\alpha}\right|\left|1+\frac{\partial u_{1}}{\partial \alpha}\right|+\left|\frac{u_{1}}{u_{1}+\alpha}\right| \kappa^{*}\left(u_{1}\right), \\
\kappa\left(b_{k}\right)= & \left|\gamma_{k-1} u_{k}\right|+\frac{\left|\gamma_{k-1} u_{k}-1\right|}{\left|b_{k}\right|}\left[\left|B_{k-1}\right|+\left|u_{k-1}\right|\left(1+\kappa^{*}\left(u_{k-1}\right)\right]+\right. \\
& +\left|\frac{\alpha}{b_{k}}\right|\left|\left(\gamma_{k-1} u_{k}-1\right) \frac{\partial u_{k-1}}{\partial \alpha}+\gamma_{k-1} u_{k}\right|, \quad k \geq 2 .
\end{aligned}
$$

where $\gamma_{k-1}$ is defined in (21).

Proof If $k=1$ then

$$
\kappa^{*}\left(u_{1}\right)=\kappa_{C}\left(u_{1}\right)+\kappa_{\mathscr{L}_{0}}\left(u_{1}\right)+\kappa_{\mu_{0}}\left(u_{1}\right)=1+\frac{|C|+\left|\mu_{0}\right|}{\left|C+\mu_{0}\right|} .
$$

Assume now that $k>1$. Then, if $i=k-1$,

$$
\begin{aligned}
& \kappa_{B_{k-1}}\left(u_{k}\right)=\frac{\left|B_{k-1}\right|+\left|u_{k-1}\right|}{\left|u_{k}\right|}\left|\frac{u_{k}}{B_{k-1}-u_{k-1}-\alpha}\right|=\frac{\left|B_{k-1}\right|+\left|u_{k-1}\right|}{\left|B_{k-1}-u_{k-1}-\alpha\right|} . \\
& \kappa_{G_{k-1}}\left(u_{k}\right)=\left|\frac{G_{k-1}}{u_{k}}\right|\left|\frac{1}{B_{k-1}-u_{k-1}-\alpha}\right|=1 .
\end{aligned}
$$

Similarly, if $i<k-1$,

$$
\begin{aligned}
& \kappa_{B_{i}}\left(u_{k}\right)=\frac{\left|B_{i}\right|+\left|u_{i}\right|}{\left|u_{k}\right|}\left|\frac{u_{k}}{B_{k-1}-u_{k-1}-\alpha}\right|\left|\frac{\partial u_{k-1}}{\partial B_{i}}\right|=\left|\frac{u_{k-1}}{B_{k-1}-u_{k-1}-\alpha}\right| \kappa_{B_{i}}\left(u_{k-1}\right) . \\
& \kappa_{G_{i}}\left(u_{k}\right)=\left|\frac{G_{i}}{u_{k}}\right|\left|\frac{u_{k}}{B_{k-1}-u_{k-1}-\alpha}\right|\left|\frac{\partial u_{k-1}}{\partial G_{i}}\right|=\left|\frac{u_{k-1}}{B_{k-1}-u_{k-1}-\alpha}\right| \kappa_{G_{i}}\left(u_{k-1}\right) .
\end{aligned}
$$

Finally,

$$
\kappa_{C}\left(u_{k}\right)=\left|\frac{C}{u_{k}}\right|\left|\frac{u_{k}}{B_{k-1}-u_{k-1}-\alpha}\right|\left|\frac{\partial u_{k-1}}{\partial C}\right|=\left|\frac{u_{k-1}}{B_{k-1}-u_{k-1}-\alpha}\right| \kappa_{C}\left(u_{k-1}\right) .
$$

The remaining two condition numbers are computed in a similar way.

These expressions lead us to the recurrence relation for $\kappa^{*}\left(u_{k}\right)$ in a straightforward way from (23). 
For $k=1$,

$$
\begin{aligned}
& \kappa_{C}\left(b_{1}\right)=\left|\frac{C}{b_{1}}\right|\left|\frac{u_{1}}{C+\mu_{0}}\right|, \quad \kappa_{\alpha}\left(b_{1}\right)=\left|\frac{\alpha}{b_{1}}\right|\left|1+\frac{\partial u_{1}}{\partial \alpha}\right| . \\
& \kappa_{\mathscr{L}_{0}}\left(b_{1}\right)=\left|\frac{u_{1}}{b_{1}}\right|, \quad \kappa_{\mu_{0}}\left(b_{1}\right)=\left|\frac{u_{1}}{b_{1}}\right|\left|\frac{\mu_{0}}{C+\mu_{0}}\right| .
\end{aligned}
$$

Assume now that $k>1$. For $i=k-1$,

$$
\begin{aligned}
& \kappa_{B_{k-1}}\left(b_{k}\right)=\frac{\left|B_{k-1}\right|+\left|u_{k-1}\right|}{\left|b_{k}\right|}\left|\frac{u_{k}}{B_{k-1}-u_{k-1}-\alpha}-1\right|=\frac{\left|B_{k-1}\right|+\left|u_{k-1}\right|}{\left|b_{k}\right|}\left|\gamma_{k} u_{k}-1\right|, \\
& \kappa_{G_{k-1}}\left(b_{k}\right)=\left|\frac{G_{k-1}}{b_{k}}\right|\left|\frac{1}{B_{k-1}-u_{k-1}-\alpha}\right|=\left|\frac{u_{k}}{b_{k}}\right| .
\end{aligned}
$$

For $i<k-1$

$$
\begin{aligned}
& \kappa_{B_{i}}\left(b_{k}\right)=\frac{\left|B_{i}\right|+\left|u_{i}\right|}{\left|b_{k}\right|}\left|\gamma_{k-1} u_{k}-1\right|\left|\frac{\partial u_{k-1}}{\partial B_{i}}\right|=\left|\gamma_{k-1} u_{k}-1\right|\left|\frac{u_{k-1}}{b_{k}}\right| \kappa_{B_{i}}\left(u_{k-1}\right), \\
& \kappa_{G_{i}}\left(b_{k}\right)=\left|\frac{G_{i}}{b_{k}}\right|\left|\gamma_{k-1} u_{k}-1\right|\left|\frac{\partial u_{k-1}}{\partial G_{i}}\right|=\left|\gamma_{k-1} u_{k}-1\right|\left|\frac{u_{k-1}}{b_{k}}\right| \kappa_{G_{i}}\left(u_{k-1}\right) .
\end{aligned}
$$

Finally,

$$
\begin{aligned}
& \kappa_{\alpha}\left(b_{k}\right)=\left|\frac{\alpha}{b_{k}}\right|\left|\left(\gamma_{k-1} u_{k}-1\right) \frac{\partial u_{k-1}}{\partial \alpha}+\gamma_{k-1} u_{k}\right|, \\
& \kappa_{C}\left(b_{k}\right)=\left|\frac{C}{b_{k}}\right|\left|\left(\gamma_{k-1} u_{k}-1\right) \frac{\partial u_{k-1}}{\partial C}\right|=\left|\gamma_{k-1} u_{k}-1\right|\left|\frac{u_{k-1}}{b_{k}}\right| \kappa_{C}\left(u_{k-1}\right) .
\end{aligned}
$$

The rest of the condition numbers can be obtained in a similar way. The result follows by (13) and (23).

The expression for $\kappa\left(g_{k}\right)$ can be found following a similar procedure.

\section{Appendix 2: Proof of Theorem 8}

It can be seen from their explicit expressions that both numbers $\kappa^{*}\left(u_{k}\right)$ and $\kappa_{S}^{*}\left(u_{k}\right)$ are larger than one. Moreover they are of the same order of magnitude as the following lemma shows.

\section{Theorem 9}

$$
\kappa_{S}^{*}\left(u_{k}\right) \leq \kappa^{*}\left(u_{k}\right) \leq 2 \kappa_{S}^{*}\left(u_{k}\right) \quad \text { for all } k \geq 1 \text {. }
$$


Proof The first inequality is clear. Notice that the second inequality is true for $k=1$. In order to prove the second inequality for $k>1$, note that

$$
\begin{aligned}
& \kappa^{*}\left(u_{k}\right)=1+\left|\gamma_{k-1} B_{k-1}\right|+\sum_{i=1}^{k-2}\left(2+\left|\gamma_{i} B_{i}\right|\right) \prod_{j=i+1}^{k-1}\left|\gamma_{j} u_{j}\right|+\prod_{j=1}^{k-1}\left|\gamma_{j} u_{j}\right|\left(2+\frac{|C|+\left|\mu_{0}\right|}{\left|C+\mu_{0}\right|}\right), \\
& \kappa_{S}^{*}\left(u_{k}\right)=1+\left|\gamma_{k-1} B_{k-1}\right|+\sum_{i=1}^{k-2}\left(1+\left|\gamma_{i} B_{i}\right|\right) \prod_{j=i+1}^{k-1}\left|\gamma_{j} u_{j}\right|+\prod_{j=1}^{k-1}\left|\gamma_{j} u_{j}\right|\left(1+\frac{|C|+\left|\mu_{0}\right|}{\left|C+\mu_{0}\right|}\right),
\end{aligned}
$$

where $\sum_{i=1}^{0} \equiv 0$ and $\sum_{i=1}^{-1} \equiv 0$, i.e., for $k=1$ the summations are not present. The result follows from the previous expressions.

It is also easy to prove that $\kappa\left(b_{k}\right)$ and $\kappa_{S}\left(b_{k}\right)$ are of the same order of magnitude for all $k \geq 1$.

Theorem 10 For $1 \leq k \leq n$,

$$
\kappa_{S}\left(b_{k}\right) \leq \kappa\left(b_{k}\right) \leq 3 \kappa_{S}\left(b_{k}\right) .
$$

Proof Again, the first inequality is obvious. In order to prove the second one take into account Theorem 9 and the fact that $1 \leq \kappa_{S}^{*}\left(u_{k}\right)$ for all $k$ to get

$$
\begin{aligned}
\kappa\left(b_{k}\right) \leq & \left|\frac{u_{k}}{b_{k}}\right|+\left|\frac{\gamma_{k-1} u_{k}-1}{b_{k}}\right|\left[\left|B_{k-1}\right|+\left|u_{k-1}\right| 3 \kappa_{S}^{*}\left(u_{k-1}\right)\right] \\
& +\left|\frac{\alpha}{b_{k}}\right|\left|\left(\gamma_{k-1} u_{k}-1\right) \frac{\partial u_{k-1}}{\partial \alpha}+\gamma_{k-1} u_{k}\right|,
\end{aligned}
$$

and the result follows.

Proving that $\kappa\left(g_{k}\right)$ and $\kappa_{S}\left(g_{k}\right)$ are of the same magnitude is not always possible. It is not true in general that $\kappa\left(g_{k}\right)$ is upper bounded by a multiple of $\kappa_{S}\left(g_{k}\right)$. However, the lemma below shows that whenever $\kappa\left(g_{k}\right)$ and $\kappa_{S}\left(g_{k}\right)$ have different orders of magnitude, then $\kappa\left(g_{k}\right)$ is bounded by $8 \kappa_{S}\left(b_{k+1}\right)$. The technical Lemma 13 will be needed to prove our claim.

Lemma 13 Let us assume that $\frac{3}{4}<\gamma_{k} u_{k}<\frac{3}{2}$ for some $k$. If $\gamma_{k} u_{k}>4\left|\gamma_{k} B_{k}\right|$, then

- if $\gamma_{k} u_{k+1}>15 / 8$ or $\gamma_{k} u_{k+1}<3 / 8$, then

$$
\frac{5}{12}<\left|\frac{u_{k+1}-1 / \gamma_{k}}{b_{k+1}}\right| \text {. }
$$

- if $3 / 8 \leq \gamma_{k} u_{k+1} \leq 15 / 8$, then

$$
\frac{1}{4}<\left|\frac{u_{k+1}}{b_{k+1}}\right| .
$$


Proof Since $\gamma_{k} u_{k}>4\left|\gamma_{k} B_{k}\right|$,

$$
-\frac{3}{8}<\gamma_{k} B_{k}<\frac{3}{8} .
$$

We consider two possible situations: $\gamma_{k}>0$ and $\gamma_{k}<0$. Let us begin by assuming that $\gamma_{k}>0$.

1. If $\gamma_{k}>0$, then $u_{k}>0$. From (26) we get

$$
-\frac{3}{8} \frac{1}{\gamma_{k}}<l_{k}+u_{k}+\alpha<\frac{3}{8} \frac{1}{\gamma_{k}} .
$$

Therefore,

$$
-\frac{15}{8 \gamma_{k}}-l_{k}<\alpha<-\frac{3}{8 \gamma_{k}}-l_{k} .
$$

Then, from (27), and taking into account that $b_{k+1}=u_{k+1}+l_{k}+\alpha$, we get the following bounds

$$
u_{k+1}-\frac{15}{8 \gamma_{k}}<b_{k+1}<u_{k+1}-\frac{3}{8 \gamma_{k}} .
$$

Notice that both bounds of $b_{k+1}$ will be positive if $u_{k+1} \gamma_{k}>15 / 8$, and both bounds will be negative if $u_{k+1} \gamma_{k}<3 / 8$.

- Let us assume that $u_{k+1} \gamma_{k}>15 / 8$, then $u_{k+1}-1 / \gamma_{k}>0$ and

$$
\frac{u_{k+1}-1 / \gamma_{k}}{u_{k+1}-\frac{3}{8 \gamma_{k}}}<\left|\frac{u_{k+1}-1 / \gamma_{k}}{b_{k+1}}\right| \text {. }
$$

Therefore,

$$
\frac{7}{12}<\frac{1}{1+\frac{5}{8} \frac{1}{u_{k+1} \gamma_{k}-1}}<\left|\frac{u_{k+1}-1 / \gamma_{k}}{b_{k+1}}\right| .
$$

- Let us assume now that $u_{k+1} \gamma_{k}<3 / 8$. Then, $u_{k+1}-1 / \gamma_{k}<0$ and

$$
\frac{-u_{k+1}+1 / \gamma_{k}}{-u_{k+1}+\frac{15}{8 \gamma_{k}}}<\left|\frac{u_{k+1}-1 / \gamma_{k}}{b_{k+1}}\right| \text {. }
$$

As a consequence,

$$
\frac{5}{12}<\frac{1}{1+\frac{7}{8} \frac{1}{1-u_{k+1} \gamma_{k}}}<\left|\frac{u_{k+1}-1 / \gamma_{k}}{b_{k+1}}\right| .
$$

- Finally, suppose that $\frac{3}{8} \leq u_{k+1} \gamma_{k} \leq \frac{15}{8}$. Then, $u_{k+1}>0$. If $b_{k+1}>0$, we get

$$
\frac{5}{4}<\frac{u_{k+1} \gamma_{k}}{u_{k+1} \gamma_{k}-\frac{3}{8}}<\left|\frac{u_{k+1}}{b_{k+1}}\right| .
$$


If $b_{k+1}<0$, then

$$
\frac{1}{4}<\frac{u_{k+1} \gamma_{k}}{-u_{k+1} \gamma_{k}+\frac{15}{8}}<\left|\frac{u_{k+1}}{b_{k+1}}\right| .
$$

2. When $\gamma_{k}<0$, a similar proof gives the same bounds.

Now we can prove Theorem 11. Let us remark that Theorem 8 is a trivial consequence of Theorems 10 and 11. Notice that, from the expressions for $\kappa\left(g_{k}\right)$ and $\kappa_{S}\left(g_{k}\right)$, and taking into account that $\kappa^{*}\left(u_{k}\right)$ and $\kappa_{S}^{*}\left(u_{k}\right)$ are of the same order of magnitude by Theorem 9 , it can easily be deduced that $\kappa\left(g_{k}\right)$ and $\kappa_{S}\left(g_{k}\right)$ have similar orders of magnitude when $u_{k} \gamma_{k}$ is not close to one. This is covered in the first two items of Theorem 11. The most difficult situation, i.e., when $u_{k} \gamma_{k}$ is close to one, is presented in the last item. Let us recall that $u_{k} \neq 0$ for all $k$ because $G_{k-1} \neq 0$ for monic Jacobi matrices corresponding to sequences of orthogonal polynomials.

Theorem 11 For $1 \leq k \leq n-1$,

1 if $u_{k} \gamma_{k}<0$, then

$$
\kappa_{S}\left(g_{k}\right) \leq \kappa\left(g_{k}\right) \leq 3 \kappa_{S}\left(g_{k}\right) .
$$

2 if $0<u_{k} \gamma_{k} \leq 3 / 4$ or $u_{k} \gamma_{k} \geq 3 / 2$, then

$$
\kappa_{S}\left(g_{k}\right) \leq \kappa\left(g_{k}\right) \leq 8 \kappa_{S}\left(g_{k}\right) .
$$

3 if $\frac{3}{4}<u_{k} \gamma_{k}<\frac{3}{2}$ for some $k$,

3.1 if $u_{k} \gamma_{k} \leq 4\left|B_{k} \gamma_{k}\right|$, then

$$
\kappa_{S}\left(g_{k}\right) \leq \kappa\left(g_{k}\right) \leq 5 \kappa_{S}\left(g_{k}\right) .
$$

3.2 if $u_{k} \gamma_{k}>4\left|B_{k} \gamma_{k}\right|$, then

(a) if $\kappa\left(g_{k}\right) \geq \frac{4}{3} u_{k} \gamma_{k}$, then

$$
\kappa_{S}\left(g_{k}\right) \leq \kappa\left(g_{k}\right) \leq 8 \kappa_{S}\left(g_{k}\right) .
$$

(b) if $\kappa\left(g_{k}\right)<\frac{4}{3} u_{k} \gamma_{k}$, then

$$
\kappa_{S}\left(g_{k}\right) \leq \kappa\left(g_{k}\right) \leq 8 \kappa_{S}\left(b_{k+1}\right) .
$$

Proof Considering the definitions of $\kappa_{(}\left(g_{k}\right)$ and $\kappa_{S}\left(g_{k}\right)$, it is easy to see that

$$
\kappa_{S}\left(g_{k}\right) \leq \kappa\left(g_{k}\right), \quad \text { for all } k .
$$

In the rest of the proof, notice that

$$
\gamma_{k} \delta_{k}=\frac{B_{k}-2 u_{k}-\alpha}{B_{k}-u_{k}-\alpha}=1-\frac{u_{k}}{B_{k}-u_{k}-\alpha}=1-\gamma_{k} u_{k} .
$$

Denote $a=u_{k} \gamma_{k}$. We need to compare the quantities $|a|+2|1-a| \kappa_{S}^{*}\left(u_{k}\right)$ and $|1-a| \kappa_{S}^{*}\left(u_{k}\right)$. Note also that $\kappa_{S}^{*}\left(u_{k}\right) \geq 1$. 
1. If $a<0$ then $|a|+2|1-a|=2-3 a \leq 3(1-a)$, and hence

$$
|a|+2|1-a| \kappa_{S}^{*}\left(u_{k}\right) \leq(|a|+2|1-a|) \kappa_{S}^{*}\left(u_{k}\right) \leq 3|1-a| \kappa_{S}^{*}\left(u_{k}\right),
$$

so $\kappa\left(g_{k}\right) \leq 3 \kappa_{S}\left(g_{k}\right)$.

2. If $0 \leq a \leq 3 / 4$, then $|a|+2|1-a|=2-a$, so

$$
|a|+2|1-a| \kappa_{S}^{*}\left(u_{k}\right) \leq(|a|+2|1-a|) \kappa_{S}^{*}\left(u_{k}\right) \leq 8|1-a| \kappa_{S}^{*}\left(u_{k}\right),
$$

and therefore $\kappa\left(g_{k}\right) \leq 8 \kappa_{S}\left(g_{k}\right)$.

3. If $a \geq 3 / 2$ then $|a|+2|1-a|=3 a-2 \leq 5(a-1)$, so

$$
|a|+2|1-a| \kappa_{S}^{*}\left(u_{k}\right) \leq(|a|+2|1-a|) \kappa_{S}^{*}\left(u_{k}\right) \leq 5|1-a| \kappa_{S}^{*}\left(u_{k}\right),
$$

and $\kappa\left(g_{k}\right) \leq 5 \kappa_{S}\left(g_{k}\right)$.

3.1 If $\frac{3}{4}<\gamma_{k} u_{k}<\frac{3}{2}$ and $u_{k} \gamma_{k} \leq 4\left|B_{k} \gamma_{k}\right|$ then, taking into account the expressions for $\kappa\left(g_{k}\right)$ and $\kappa_{S}\left(g_{k}\right)$, the result follows.

3.2 If $\frac{3}{4}<\gamma_{k} u_{k}<\frac{3}{2}$ and $u_{k} \gamma_{k}>4\left|B_{k} \gamma_{k}\right|$ then, the condition $\kappa\left(g_{k}\right) \geq \frac{4}{3} u_{k} \gamma_{k}$ implies

$$
\begin{aligned}
8 \kappa_{S}\left(g_{k}\right) & \geq 4\left\{\left|\gamma_{k}\right|\left[\left|B_{k}\right|+\left|\delta_{k}\right| \kappa\left(u_{k}\right)\right]+\left|\gamma_{k} \alpha\right|\left|1-\frac{\delta_{k}}{u_{k}} \frac{\partial u_{k}}{\partial \alpha}\right|\right\} \\
& \geq 4 \kappa\left(g_{k}\right)-4\left|u_{k} \gamma_{k}\right|>\kappa\left(g_{k}\right) .
\end{aligned}
$$

On the other hand, if $\kappa\left(g_{k}\right)<\frac{4}{3} u_{k} \gamma_{k}$ and $u_{k+1} \gamma_{k}>\frac{15}{8}$ or $u_{k+1} \gamma_{k}<\frac{3}{8}$, then by Lemma 13

$$
\kappa_{S}\left(b_{k+1}\right) \geq\left|\frac{u_{k+1}-1 / \gamma_{k}}{b_{k+1}}\right|\left|u_{k} \gamma_{k}\right|>\frac{5}{12}\left|u_{k} \gamma_{k}\right|,
$$

which implies

$$
\kappa\left(g_{k}\right)<\frac{16}{5} \kappa_{S}\left(b_{k+1}\right) .
$$

When $\kappa\left(g_{k}\right)<\frac{4}{3} u_{k} \gamma_{k}$ and $\frac{3}{8} \leq u_{k} \gamma_{k} \leq \frac{15}{8}$, by Lemma 13

$$
\kappa_{S}\left(b_{k+1}\right) \geq \frac{1}{4}
$$

Moreover, since $u_{k} \gamma_{k}<\frac{3}{2}, \kappa\left(g_{k}\right)<\frac{4}{3} u_{k} \gamma_{k} \leq 2$, which implies

$$
\kappa\left(g_{k}\right) \leq 8 \kappa_{S}\left(b_{k+1}\right)
$$

\section{References}

1. Bueno, M.I., Marcellán, F.: Darboux transformation and perturbation of linear functionals. Linear Algebra Appl. 384, 215-242 (2004)

2. Buhmann, M., Iserles, A.: On orthogonal polynomials transformed by the QR algorithm. J. Comp. Appl. Math. 43, 117-134 (1992) 
3. Chaitin-Chatelin, F., Fraysse, V.: Lectures on Finite Precision Computations. SIAM, Philadelphia (1996)

4. Chihara, T.S.: An Introduction to Orthogonal Polynomials. Gordon and Breach, New York (1957)

5. Elhay, S., Kautsky, J.: Jacobi matrices for measures modified by a rational factor. Numer. Algorithms 6, 205-227 (1994)

6. Faddeev, D.K., Faddeeva, V.N.: Computational Methods of Linear Algebra. Transl. from Russian. W.H. Freeman, San Francisco (1963)

7. Galant, D.: An implementation of christoffel's theorem in the theory of orthogonal polynomials. Math. Comput. 25, 111-113 (1971)

8. Galant, D.: Algebraic methods for modified orthogonal polynomials. Math. Comput. 59, 541-546 (1992)

9. Gautschi, W.: Minimal solutions of three-term recurrence relations and orthogonal polynomials. Math. Comput. 36(154), 547-554 (1981)

10. Gautschi, W.: Orthogonal polynomials: computations and approximation. Oxford University Press, New York (2004)

11. Gautschi, W.: Computational aspects of three-tem recurrence relations. SIAM Rev. 9(1), 24-82 (1967)

12. Gautschi, W.: The interplay between classical analysis and (numerical) linear algebra- a tribute to Gene H. Golub. Electron. Trans. Numer. Anal. 13, 119-147 (2002)

13. Geronimus, Y.L.: On the polynomials orthogonal with respect to a given number sequence and a theorem. In: Hahn, W., Nauk, I.A. (eds.), vol. 4, pp. 215-228 (1940, in Russian)

14. Geronimus, Y.L.: On the polynomials orthogonal with respect to a given number sequence. Zap. Mat. Otdel. Khar'kov. Univers. i NII Mat. i Mehan., 17, 3-18 (1940)

15. Golub, G.H., Kautsky, J.: Calculation of gauss quadratures with multiple free and fixed knots. Numer. Math. 41, 147-163 (1983)

16. Grünbaum, F.A., Haine, L.: Orthogonal polynomials satisfying differential equations: the role of the Darboux transformation. In: CRM Proceedings and Lecture Notes, American Mathematical Society, vol 9, pp. 143-154. American Mathematical Society, Providence (1996)

17. Grünbaum, F.A., Haine, L.: Bispectral Darboux transformations: an extension of the krall polynomials. Internat. Math. Res. Notices 8, 359-392 (1997)

18. Grünbaum, F.A., Haine, J., Horozov, E.: Some functions that generalize the Krall-Laguerre polynomials. J. Comp. Appl. Math. 106, 271-297 (1999)

19. Higham, N.J.: Accuracy and Stability of Numerical Algorithms, 2nd edn. SIAM, Philadelphia (2002)

20. Matveev, V.B., Salle, M.A.: Differential-difference evolution equations. II (Darboux transformation for the Toda lattice). Lett. Math. Phys. 3, 425-429 (1979)

21. Rutishauser, H.: Der Quotienten-differenzen-algorithmus. Birkhäuser, Boston (1957)

22. Spiridonov, V., Vinet, L., Zhedanov, A.: Spectral transformations, self-similar reductions and orthogonal polynomials. J. Phys. A: Math. \& Gen. 30, 7621-7637 (1997)

23. Spiridonov, V., Zhedanov, A.: Discrete Darboux transformations, discrete time Toda lattice and the Askey-Wilson polynomials. Methods and Appl. Anal. 2, 369-398 (1995)

24. Uvarov, V.B.: The connection between systems of polynomials that are orthogonal with respect to different distribution functions. Ah. Vychisl. Matem. i Mat. Fiz. 9, 1253-62 (1969)

25. Yoon, G.J.: Darboux transformation and orthogonal polynomials. Bull. Korean Math. Soc. 39(3), 359-376 (2002) 